\title{
A micromechanical interpretation of the temperature dependence of Beremin model parameters for french RPV steel
}

\author{
Jean-Philippe MATHIEU* \\ Material and Mechanics of Components Department \\ EDF RED - Les Renardières - F-77818 Moret-sur-Loing \\ Karim INAL \\ Ecole des Mines de Saint Etienne - CMP G. Charpak - F-13541 Gardanne \\ Sophie BERVEILLER \\ ENSAM CER de Metz - LPMM - F-57078 Metz CEDEX 03 \\ Olivier DIARD \\ EDF - CNPE de Penly - F-76370 Neuville-Lès-Dieppe
}

\begin{abstract}
Local approach to brittle fracture for low-alloyed steels is discussed in this paper. A bibliographical introduction intends to highlight general trends and consensual points of the topic and evokes debatable aspects. French RPV steel 16MND5 (equ. ASTM A508 Cl.3), is then used as a model material to study the influence of temperature on brittle fracture. A micromechanical modelling of brittle fracture at the elementary volume scale already used in previous work is then recalled. It involves a multiscale modelling of microstructural plasticity which has been tuned on experimental inter-phase and inter-granular stresses heterogeneities measurements. Fracture probability of the elementary volume can then be computed using a randomly attributed defect size distribution based on realistic carbides repartition. This defect distribution is then deterministically correlated to stress heterogeneities simulated within the microstructure using a weakest-link hypothesis on the elementary volume, which results in a deterministic stress to fracture. Repeating the process allows to compute Weibull parameters on the elementary volume. This tool is then used to investigate the physical mechanisms that could explain the already experimentally observed temperature dependence of Beremin's parameter for 16MND5 steel. It is showed that, assuming that the hypothesis made in this work about cleavage micromechanisms are correct, effective equivalent surface energy (i.e surface energy plus plastically dissipated energy when blunting the crack tip) for propagating a
\end{abstract}


crack has to be temperature dependent to explain Beremin's parameters temperature evolution.

Key words:

low alloyed steel, microstructure, mechanical behaviour, micromechanical modelling, mean field model, aggregate, local approach to fracture, cleavage

\section{Introduction}

At the microscopic scale, brittle fracture of low-alloyed steel is usually interpreted as an intra-granular cleavage [1]. Criteria and micro-mechanisms of fracture are still discussed, but a certain consensus can be established about the essential role of plasticity (i.e. of the dislocations movements) and about the required presence of micro-defects to cause cleavage fracture. An historical review of these aspects can be found in [2].

At the macroscopic scale, local approach [3], and global approach [4] to fracture both rely on an interpretation of the micromechanisms of fracture consequences on the macroscopic fracture behaviour, with good results $[5,6]$, but also with some limitations concerning the transferability of the fitted material parameters for different temperatures and/or geometries.

Concerning the local approach, these limitations can be considered as inherent to the fitting procedures (sampling or statistical methodology issues) and to the weaknesses of some of the Beremin's hypothesis [7]. However, a general trend has appeared in the last decades that consists in an empirical enrichment of the local approach fracture criterion originally proposed by the Beremin work-group [8-10].

French RPV steel 16MND5, and other similar steels widely used in the nuclear industry, are considered to have a brittle fracture which is governed by a Weibull law :

$$
P_{f}=1-\exp \left(-\frac{\sigma_{w}}{\sigma_{u}}\right)^{m}
$$

, where $m$ represents the statistical scatter, and $\sigma_{u}$ represents the mean stress

* Corresponding author

Email addresses: jean-philippe.mathieu@edf.fr (Jean-Philippe MATHIEU), inal@emse.fr (Karim INAL), sophie.berveiller@metz.ensam.fr (Sophie BERVEILLER), olivier.diard@edf .fr (Olivier DIARD). 
needed for fracture, $\sigma_{w}$ being the Weibull stress [3] representing an average of the first principal stress over the plastically activated zone.

Another threshold parameter $\left(\sigma_{w}^{\min }\right)$ is sometimes introduced to improve the experimental fitting results, so that equation 1 becomes :

$$
P_{f}=1-\exp \left(-\frac{\sigma_{w}-\sigma_{w}^{m i n}}{\sigma_{u}}\right)^{m}, \text { when } \sigma_{w} \leq \sigma_{w}^{m i n}
$$

, like found in $[11,12]$ but the best results are obtained considering a temperature dependence of the Weibull parameters that has been widely observed and taken into account in recent work $[13,11,14]$, and considering damage mechanisms [15].

But the precise description of the physical mechanisms leading to this temperature dependence is still to be understood. Lin already captured the complexity of the topic twenty years ago [16]: each phenomenon involved in brittle fracture is somehow governed by temperature. Therefore, a macroscopic observation of the temperature dependence does not guaranty a correct interpretation of the way temperature influences brittle fracture parameters at the micromechanical scale, because cross effects can be difficult to distinguish.

Back to the micromechanisms of brittle fracture of steels, three steps are generally considered. The initiation of a micro-defect is the first step generally related to a second-phase particle failure and/or to plasticity mechanisms. Its propagation to the contigous lattice is then considered as the second step, which is sometimes followed by the last step, consisting in the microstructural barriers crossing. These steps are not critical all at the same time, depending on material characteristics, and on thermomechanical loading.

This leads to the actual questioning about the influence of temperature on fracture stress statistical scatter. In the context of low-alloyed steel brittle fracture where propagation of a micro-crack is generally considered as the critical event for fracture, Wallin proposed to explain this temperature dependence [4] by the fact that the effective ferritic lattice toughness at a temperature different from 0K, is composed of two terms : the Surface Energy (SE), which is the athermal part related to lattice brittle toughness, and a plasticity related component, which represents the plastic work needed for mechanisms opposed to crack propagation, like plastic blunting at the crack tip. The plasticity related work component being expressed as proportional to the Peierls-Nabarro driving force.

To the authors knowledge, although this idea seems quite seducing due to its micromechanical origin, it is still discussed, and it hasn't been experimentally proven due to the inherent difficulty of its observation. On the other hand, 
other parameters were used in the brittle fracture macroscopic models : plastic correction first proposed in the Beremin work group approach [3], was used again in the last WST model development [17], and the introduction of a dependence to the yield stress, proposed by Bordet [7], can be seen as an indirect way to take this temperature dependence of fracture into account.

Methodologies were also thoroughly developed during the last decade that allow to investigate the microstructural heterogeneities effects on the local heterogeneities of the mechanical fields during plastic straining [18]. These methodologies allow a better micromechanical representation of materials behaviour but also usually lead to a better description of the behaviour at the macroscopic scale. Moreover they can also provide really accurate information on the mechanical fields repartition: between metallurgical phases in case of composite materials representation or between the crystallographic orientations of a polycrystalline material. The recent availability of large performance computing also enabled explicit microstructure morphology representation [19] allowing to go further than the Mean Field (MF) models about intra-phase fields repartition description. These methods allowed a more precise local phenomena representation : grain boundaries and neighboring effects and the induced local stress repartition can be fairly well described, which can be considered as essential when realistic defect repartition representation are to be confronted deterministically to stress heterogeneities at the microscopic scale. These approaches already proved their relevance for comprehension of different phenomena, like damage of austenitic steels [20] or intergranular damage modelling [21] of zirconium alloys.

Previous work $[22,23]$ emphasized experimentally those stress heterogeneities inside the 16MND5 during plastic straining at different low temperatures. A complex multiscale modelling was set up in order to reproduce those heterogeneities at the scale of an Elementary Volume (EV) [2], and efforts were made to compute fracture probability based on a micromechanical criterion $[24,25]$ applied at the microscale of this multiscale modelling. Thus, this micromechanical tool allowed to study separately several parameters variation effect on the computed fracture probability of an EV.

This allowed recently to demonstrate that the first principal stress may not be the best governing stress parameter for local fracture criterion, as the local stress triaxiality state also appears to be an important parameter when considering the crystallographic event of cleavage [26]. The explanation given in this paper is that for a given first principal stress inside an EV, a higher stress triaxiality induces a different statistical repartition of stresses on cleavage planes likely to change the probability that a "large enough" defect meets a "loaded enough" zone of the material. It was thus showed that stress triaxiality has an obvious effect on Weibull parameter $m$, so that an increase in stress triaxiality also increases the identified $m$ value. 
the present work will use this micromechanical tool and its precise description of stresses repartition in 16MND5 french RPV during plastic straining to discuss the temperature effect on the Weibull parameters describing the fracture of an EV. Low temperature $\left(-150^{\circ} \mathrm{C} ;-60^{\circ} \mathrm{C}\right)$ will be used as it is a convenient way to study the steel within its brittle behaviour domain.

\section{Multiscale modelling of material behaviour}

\subsection{Metallurgical bases}

16MND5 steel exhibits the microstructure of a tempered bainite [27]. Its main constituents are a ferritic matrix which is reinforced with a distibution of carbides. Observations of the material by Scanning Electron Microscopy can be found on figure 1. With this technique, ferrite appears as the dark phase and carbides appear as white on figures 1.a and 1.c.

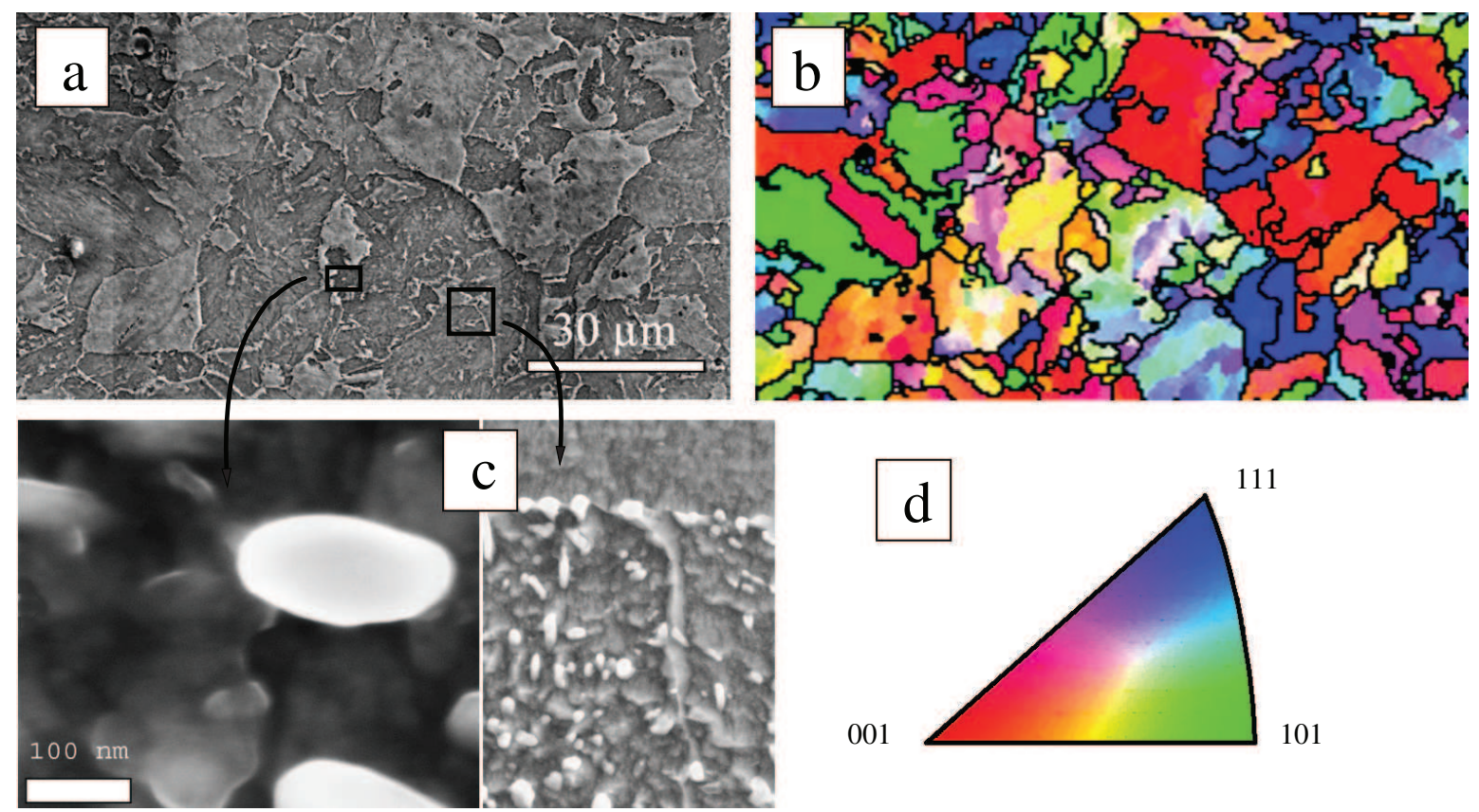

Fig. 1. Cross observation of the same sample zone by different electronic microscopy techniques. a) SEM, b) EBSD observation of the lath packets, c) Field emission gun SEM observation. d) Color legend for EBSD (indicated orientations of crystallites is relative to the horizontal axis of this paper, misorientations between contiguous domains highers than $10^{\circ}$ are highlighted in black).

Two main metallurgical sources for mechanical heterogeneities were highlighted in a previous experimental work on this material. One may want to consult experimental specific results and characterization overview in [2]. In 
short, these effects were separated and named as a "composite" one and a "polycrystalline" one.

The composite effect is related to the hardening effect of carbides (the role of carbides in fracture being discussed later). This effect is well known : as a harder phase, carbides will support a lot more stress than the ferritic matrix, and this matrix will need to accommodate strain.

The polycrystalline effect is also quite usual in structural steels, and is related to difference of lattice orientation between ferrite lath packets : this will induce different apparent behaviours of lath packets according to their orientation. The so-called "lath packet" microstructure results from the material thermal process. Figure 1.b exhibits this lath packet morphology.

During in-situ tensile testing at $-150^{\circ} \mathrm{C}$, mean stress in ferritic matrix determined by use of X-ray diffraction methods was found to be $150 \mathrm{MPa}$ lower than the applied stress [2] (composite effect). Inside the ferritic matrix, differences in residual stresses between misoriented ferrite lath packets can reach $100 \mathrm{MPa}$ (first order polycrystalline effect). Surface relief observations inside SEM during straining also indicates that there are high heterogeneities inside lath packets, particularly near interfaces, due to strain accommodation between differently oriented lath packets. All these results highlight a complex stress repartition at the microscopic scale that the modelling part of this work will have to take into account.

\subsection{Strategy}

Different scales will be represented in the modelling part of this work. The smallest scale involved will use a micromechanical representation of the elastoplastic behaviour of the ferritic phase, based on crystallographic gliding systems. At a higher scale, a MF model will be involved in the composite effect representation. The resulting behaviour, representing both ferrite and carbides will then be implemented in FE multicrystalline aggregate that stands for representing the polycrystalline effect of the lath packets.

These aspects are represented in figure 2. One can observe that a specific attention is paid to the relation between real microstructural heterogeneities and modelling features. The following sections aim to describe precisely each of those modelling steps. 


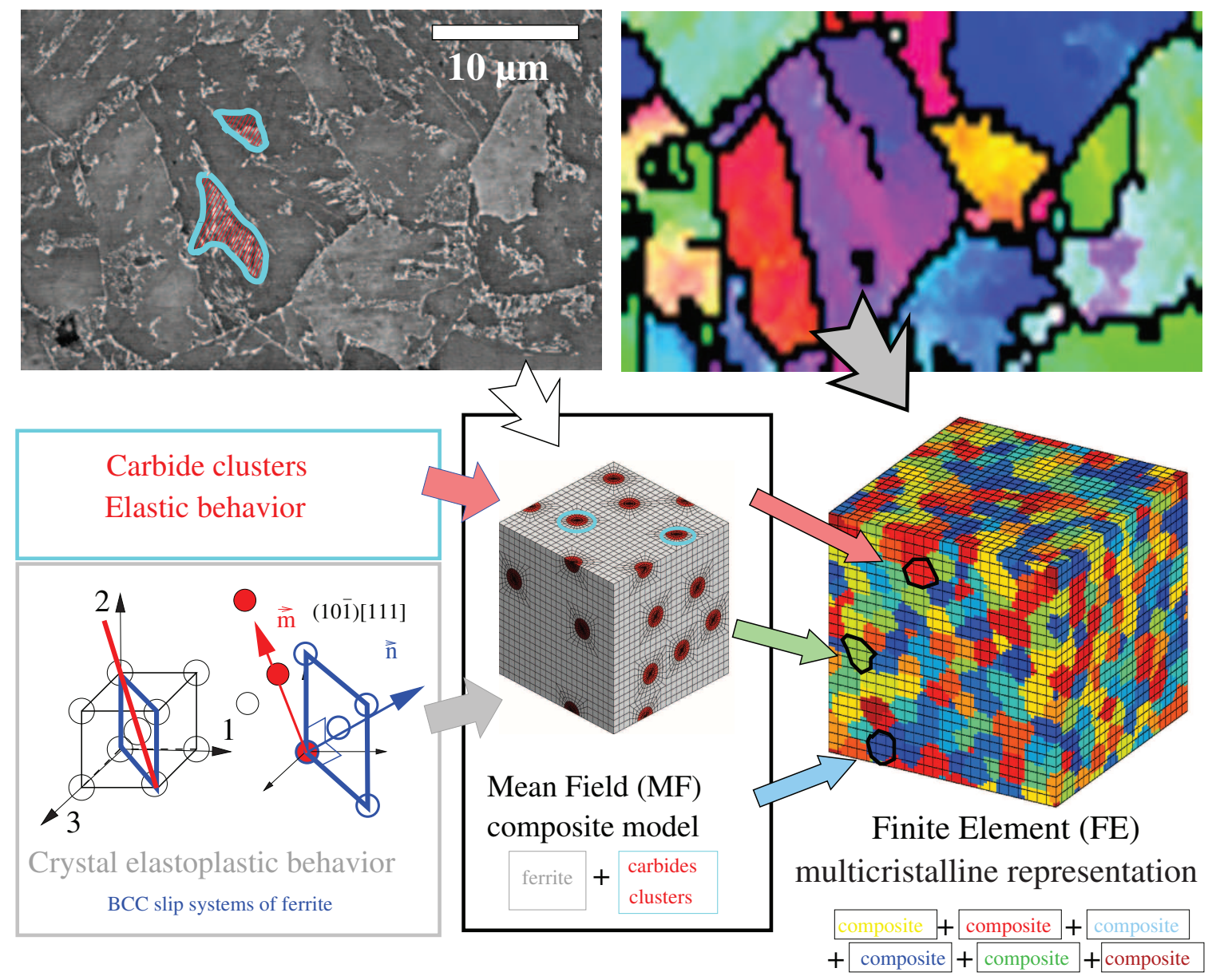

Fig. 2. Representation of the material multiscale modelling strategy, with schematic links to the microstructures features, and representation of the scale transitions.

\subsection{Ferrite single crystal behaviour}

Since no loading rate effect is to be taken into account in this study, modelling of ferrite behaviour will use an elastoplastic formulation. Thus, ferrite elastoplastic strain $£$ will be classically expressed as the sum of a plastic strain $\varepsilon^{p}$ and of an elastic one $\xi^{e}$ :

$$
\varepsilon=\varepsilon^{e}+\varepsilon^{p}
$$

One should notice that this equation and all the following until equation 9 refer to the ferritic behaviour. They are thus expressed in the local lattice crystallographic frame. The elastic part follows the generalized Hooke's law within small deformation assumption :

$$
\sigma=\underset{\approx}{\mathbf{C}}:{\underset{\varepsilon}{e}}^{e}
$$


where $\underset{\approx}{\mathbf{C}}$ represent the isotropic elastic moduli tensor, which can easily be deduced from classical elastic constants $E$ and $\nu$. Plastic gliding in ferrite's iron BCC lattice is considered to be possible on two crystallographic slip systems families, $<111>\{110\}$ and $<111>\{112\}$. Cubic symmetry of the the lattice thus enables 24 gliding systems, so that the plastic strain rate tensor $\dot{\check{\varepsilon}}^{p}$ will be the sum of the shear strain rates $\dot{\gamma}^{g}$ over all the activated slip systems $g:$

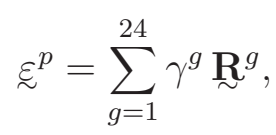

with $\underset{\sim}{\mathbf{R}}{ }^{g}$ representing a geometrical projection tensor, depending of the normal to the slip system $\underline{\mathbf{n}}^{g}$ and of its direction of gliding $\underline{\mathbf{m}}^{g}$ :

$$
\mathbf{R}^{g}=1 / 2\left(\underline{\mathbf{m}}^{g} \otimes \underline{\mathbf{n}}^{g}+\underline{\mathbf{n}}^{g} \otimes \underline{\mathbf{m}}^{g}\right) .
$$

One can find an illustration of $\underline{\mathbf{n}}^{g}$ and $\underline{\mathbf{m}}^{g}$ vectors in the figure 2 . The shear strain rates $\dot{\gamma}^{g}$ of a slip system $g$ depends on the difference between the actual Resolved Shear Stress (RSS) $\tau^{g}$ and the actual critical RSS $\tau_{c}{ }^{g}$ :

$$
\begin{aligned}
& \dot{\gamma}^{g}=\left(\frac{\left|\tau^{g}\right|-\tau_{c}^{g}}{K}\right)^{n} \cdot \operatorname{sign}\left(\tau^{g}\right), \\
& \text { with } \operatorname{sign}(a)=\left\{\begin{array}{l}
a /|a| \text { for } a \neq 0, \\
a \text { for } a=0,
\end{array}\right.
\end{aligned}
$$

so that $\tau_{c}^{g}$ represents the actual limit for plastic gliding triggering. The RSS $\tau^{g}$ being the projection of the stress tensor $\sigma$ on the considered slip system $g$ :

$$
\tau^{g}=\mathbf{R}^{g}: \underset{\sim}{\sigma}
$$

and the critical RSS evolving in accordance with :

$$
\tau_{c}^{g}=\tau_{c_{0}}^{g}+Q^{g} \sum_{h=1}^{24} h^{g h} \cdot\left(1-e^{-b^{g} \cdot \gamma_{c u m}{ }^{h}}\right) .
$$

This last equation describes the hardening of the ferritic matrix. It is inspired from the micromechanical isotropic hardening proposed in [28]. The phenomenological expression of hardening with an exponential term, allowing hardening saturation, was first proposed in [29]. $\tau_{c_{0}}^{g}$ is the initial critical RSS on the considered system $g$, which can be seen as the limit for micro-plasticity triggering for the considered system. $Q^{g}$ and $b^{g}$ are parameters of the isotropic 
hardening formulation and $h^{g h}$ is the hardening matrix, describing the interactions between slip systems (i.e. relative hardening effect of the system $h$ on the system $g$ ). One should notice that this formulation is considered as elastoviscoplastic, but a choice of great value for parameters $K$ and $n$ (respectively 12 and 5) annihilates any viscous effect, leading to a quasi-elastoplastic behaviour within standard strain rates considered in this work $\left(\dot{\varepsilon} \in\left[10^{-6} ; 1\right]\right)$ [2].

\subsection{Ferrite-carbide composite representation}

Previous work on micromechanical modelling of this material highlighted difficulties of classical MF models to take the hardening effect of carbides into account for realistic volume fraction. Maximum carbide volume fraction can be simply estimated around $2 \%$ in the material due to its chemical composition [2]. Those estimates where recently experimentally confirmed [30]. However, much higher carbide volume fraction had to be considered in other work [22] by means of the Mori-Tanaka [31] approach.

A particular aspect of carbides repartition was evidenced in the studied material that may explain this difference. Apart from bigger carbides like those observed near the former austenitic joints [11], smaller carbides like those observed in figure 1 were found gathered in carbide clusters, which is illustrated in figure 2. This is believed to be the explanation why Mori-Tanaka approach, can't report the stress difference experimentally observed. Theses small carbides in clusters are small and close enough to increase hardening comparatively to bigger ones by a Orowan hardening effect for the same volume fraction. Mori-Tanaka approach, which implicitely supposes that inclusions are regularly diluted in the matrix, can't report such effect.

In order to take those carbide clusters effect into account correctly with a realistic volume fraction, another MF model will be used. Since classical models were found inefficient, it was chosen to consider clusters (which means close to each other carbides plus ferrite embedded) as the hard phase and to use a model with ability to increase phenomenologically the interphase hardening. The so-called " $\beta$ model" [32] was chosen. This model allows to consider the behaviour of a Representative Volume Element (RVE ; i.e. the relation between global stress and strain, ${\underset{\sim}{\mathbf{S}}}^{R V E}$ and $\underset{\sim}{\mathbf{E}^{R V E}}$ ) as a function of its phases behaviours (relation between each phase $n$ stress and strain, $\sigma^{n}$ and $\varepsilon^{n}$ ). One should notice that, in this paper, the name RVE indicates a volume related to mechanical behaviour estimation considerations, and is to be distinguished from the Elementary Volume EV, which is related to local approach to fracture. 
Like most of the MF models, $\beta$ model is based on the Hill-Mandel lemma, which allows to express the stress and strain states inside a RVE as the average of stresses and strains of every phases $n$ in this RVE :

$$
\text { and }\left\{\begin{array}{l}
\underset{\sim}{\mathbf{E}}=<\underset{\sim}{\varepsilon}>_{R V E}=<\mathfrak{\sim}^{n}>_{R V E} \\
\underset{\sim}{\mathbf{S}}=<\underset{\sim}{\mathbf{S}}>_{R V E}=<\sigma^{n}>_{R V E}
\end{array}\right.
$$

The operation $\langle a\rangle_{V}$ stands for the average of $a$ over the domain $V$. In our case, the two considered phases will be the ferritic matrix, and the carbide clusters. One should notice that the carbide clusters phase will be considered as elastic. For any phase $n$ the localization rule of the $\beta$ model will be expressed as following:

$$
\sigma^{n}=\underset{\sim}{\mathbf{S}}+2 \mu(1-\beta)\left(\underset{\sim}{\mathbf{B}}-{\underset{\sim}{\beta}}^{n}\right),
$$

with $\beta$ depending on the inclusion's morphology represented (see section 3), and ${\underset{\sim}{n}}^{n}$ being a tensorial value representing the hardening of the phase $n$, which evolves according to :

$$
{\underset{\sim}{\beta}}^{n}=\underset{\sim}{\dot{\varepsilon}_{p}^{n}}-D^{n}\left(\dot{\varepsilon}_{p}^{n, \text { Mises }}\left(\underset{\sim}{\beta^{n}}-\delta^{n}{\underset{\sim}{\varepsilon}}_{p}^{n}\right)\right)
$$

$\varepsilon^{\text {Mises }}$ represents the von Mises equivalent strain which differs somehow from its stress counterpart :

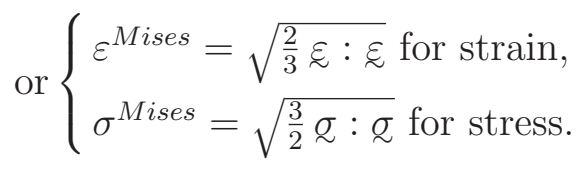

$D^{n}$ and $\delta^{n}$ are adjustable coefficients of the $\beta$ model. They have to be tuned on FE computations that use the morphology that one wants to represent. This will be discussed further in section 3. $\underset{\sim}{\mathrm{B}}$ represents the average of $\underset{\sim}{\beta^{n}}$ over the RVE :

$$
\underset{\sim}{\mathbf{B}}=\left\langle{\underset{\sim}{n}}^{n}\right\rangle_{R V E},
$$

Since carbide clusters behaviour is considered as elastic, no interphase hardening is associated to them: $D=\delta=0$. 


\subsection{Multicrystalline scale transition}

The presented MF model is then implemented at each Integration Point (IP) on a regularly meshed 3D aggregate, using the Euler angles convention to represent the BCC lattice orientation inside each lath packet. A specificity of the FE code ZeBuLoN [33] will be used: its ability to attribute a different behaviour to each IP of an element will make lath packets representation easier. An example of aggregate is presented in figure 3. It is composed of 200 grains (groups of IP), and will be used for fracture modelling (section 4), in opposition to the aggregate of figure 2, which will be used for behaviour calibration. Algorithms for grain microstructure generation were first proposed in [34] and were introduced in $\mathrm{ZeBuLoN} \mathrm{FE}$ code and generalized to 3D microstructure $[35,36]$.

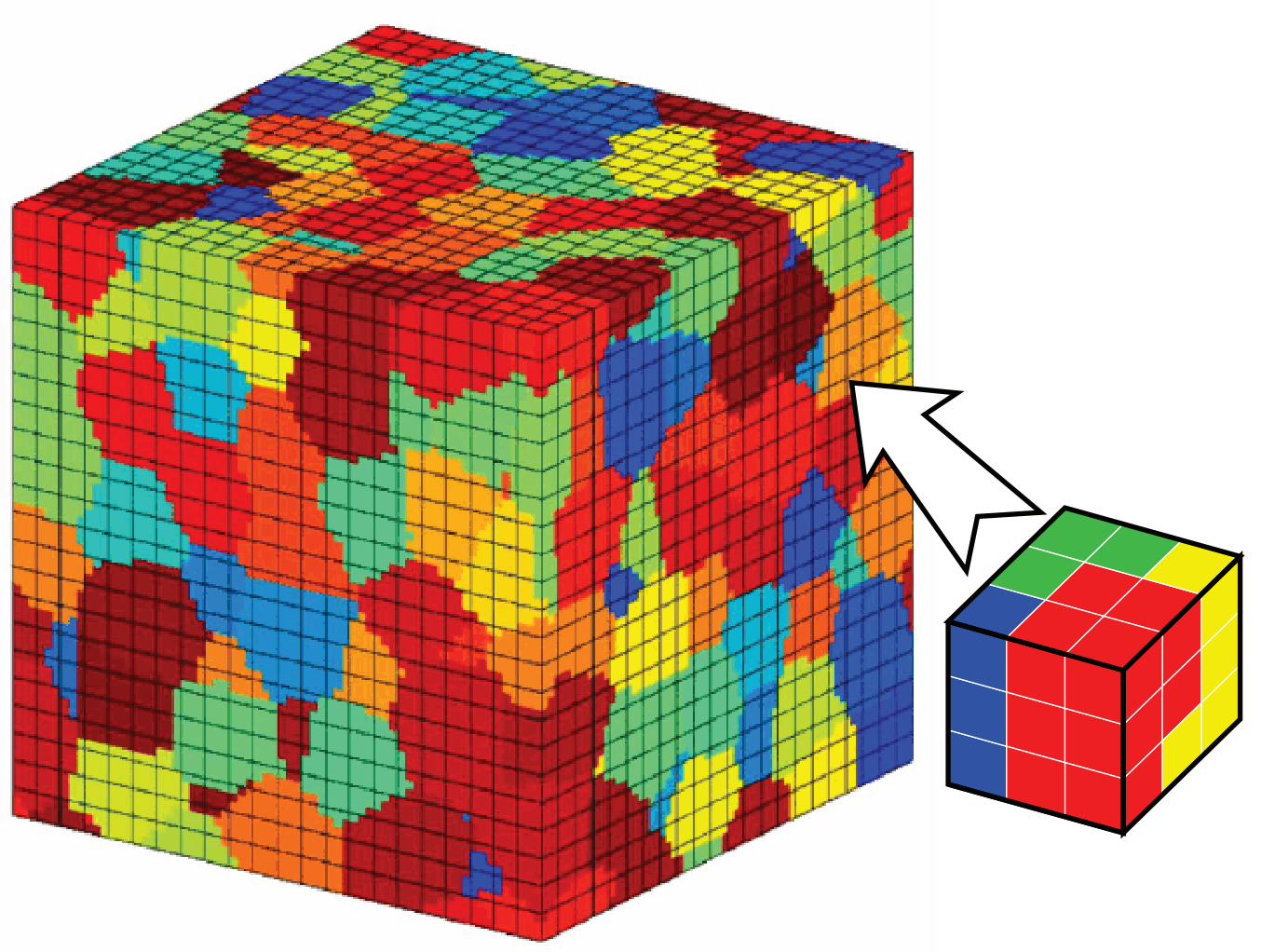

Fig. 3. A grain microstructure generated using FE code ZeBuLoN. Elements are cubic with quadratic shape functions. They include 27 IP. IP are represented as plain subdivisions of the element in this figure and the following. 


\section{Calibration and validation of plasticity modelling}

\subsection{Modelling choices}

\subsubsection{Ferrite and carbide clusters behaviours}

Classical values are attributed to ferrite elasticity coefficients : The Young modulus $E$ will be of $210000 \mathrm{MPa}$ and the Poisson ratio $\nu$ will have a value of 0.3. Since carbide clusters are supposed to be elastic, they will share the same elasticity coefficients : carbides coefficients being quite close to ferrite's ones [37].

Parameters of elastoviscoplastic behaviour, in equation 7 , are chosen as to annihilates the viscous effect [2]. Their values are respectively $K=5$ and $n=12$.

The interaction matrix $h^{g h}$ has been inspired from previous work about ferritic steels $[38,39]$. No possibility were found when this work was done to adjust those parameters on sub-scale simulations in ferrite. Thus, it was chosen to rely on usual knowledge about hardening mechanisms in ferrite [40,41]. Two gliding system families were considered : $<111>\{110\}$ and $<111>\{112\}$, they will be named respectively $F_{110}$ and $F_{112}$ in the following. Four different coefficients where introduced in the interaction matrix $\left(24^{2}\right.$ components) with this repartition :

- $h_{1}$ : interactions of families $F_{110}$ with $F_{112}$, and $F_{110}$ with itself, with systems sharing neither the gliding plane nor the direction;

- $h_{2}$ : interactions of families $F_{110}$ with $F_{112}$, and $F_{110}$ with itself, with systems sharing either the gliding plane or the direction;

- $h_{3}$ :interactions of family $F_{112}$ with itself, with systems sharing neither the gliding plane nor the direction.;

- $h_{4}$ :interactions of family $F_{112}$ with itself, with systems sharing either the gliding plane or the direction.

$h_{i}$ values where chosen as to support gliding on the family $F_{110}$ compared to the $F_{112}$ family, which is known to be more difficult to activate, and will thus be hardened more easily so that $h_{3}$ and $h_{4}$ are greater than $h_{1}$ and $h_{2}$. Also they are chosen in order to differentiate strong interaction between co-planar or co-linear systems (i.e. respectively systems sharing the same gliding plane or the same gliding direction), so that $h_{2}$ and $h_{4}$ are chosen greater respectively than $h_{1}$ and $h_{3}$. This leads to the following values: $h_{1}=1$., $h_{2}=1.15, h_{3}=1.2$, $h_{4}=1.4$.

That leaves only three parameters left to be calibrated for ferritic phase be- 
haviour : $Q, b$ and $\tau_{c}{ }^{g}$.

\subsubsection{Composite representation}

SEM observation [2] showed that carbide clusters and the ferrite embedded act globally as a bigger and harder entity (surface relief shows that the plastic flow lines do not cross the cluster, but circumvent it). So that in figures 2 and 4, the whole mixture inside the cyan lines can be considered as the hard phase. This figure also illustrates that carbides clusters did not show neither any preferential shape nor any preferential orientation in case of elongated clusters. Clusters will thus be considered as spherical inclusions. One should notice that the area observed in figure 4.a is quite rich in clusters for an illustration purpose.

Figure 4.b illustrates the chosen morphology representation mesh. This mesh will be used to calibrate values of $\delta$ and $D$ parameters for the model. Volume fraction of clusters was estimated to $3 \%$ thanks to microscopy observations, and was further found adequate to describe the stress difference between the ferritic phase and the bainitic one.

This modelling choice implies that a distinction is made in this work between the biggest carbide population that plays a role in fracture, and carbide clusters that are mainly considered to participate in composite effect. Distinction between the two populations is implicit (i.e. no size limit is established in this modelling for a carbide to play one role or the other), and this will be discussed further in section 4 .

Since a spherical morphology has been chosen for inclusions, $\beta$ can be simply expressed thanks to isotropic elasticity of both phases and to the spherical morphology previously considered for clusters:

$$
\beta=\frac{2(4-5 \nu)}{15(1-\nu)} .
$$

$\beta$ model calibration was made on a single arbitrary chosen ferrite orientation $\left(18^{\circ}, 12^{\circ}, 82^{\circ}\right)$. Results of further calibration were not found to be influenced by this choice.

\subsubsection{Multicrystalline aggregate}

Multicrystalline aggregate presented in figure 2 was used for this part of the work : it comprises 1000 lath packets which was found adequate for macroscopic behaviour calibration. No macroscopic crystallographic texture was found in the material so that it was chosen to attribute orientations randomly. 

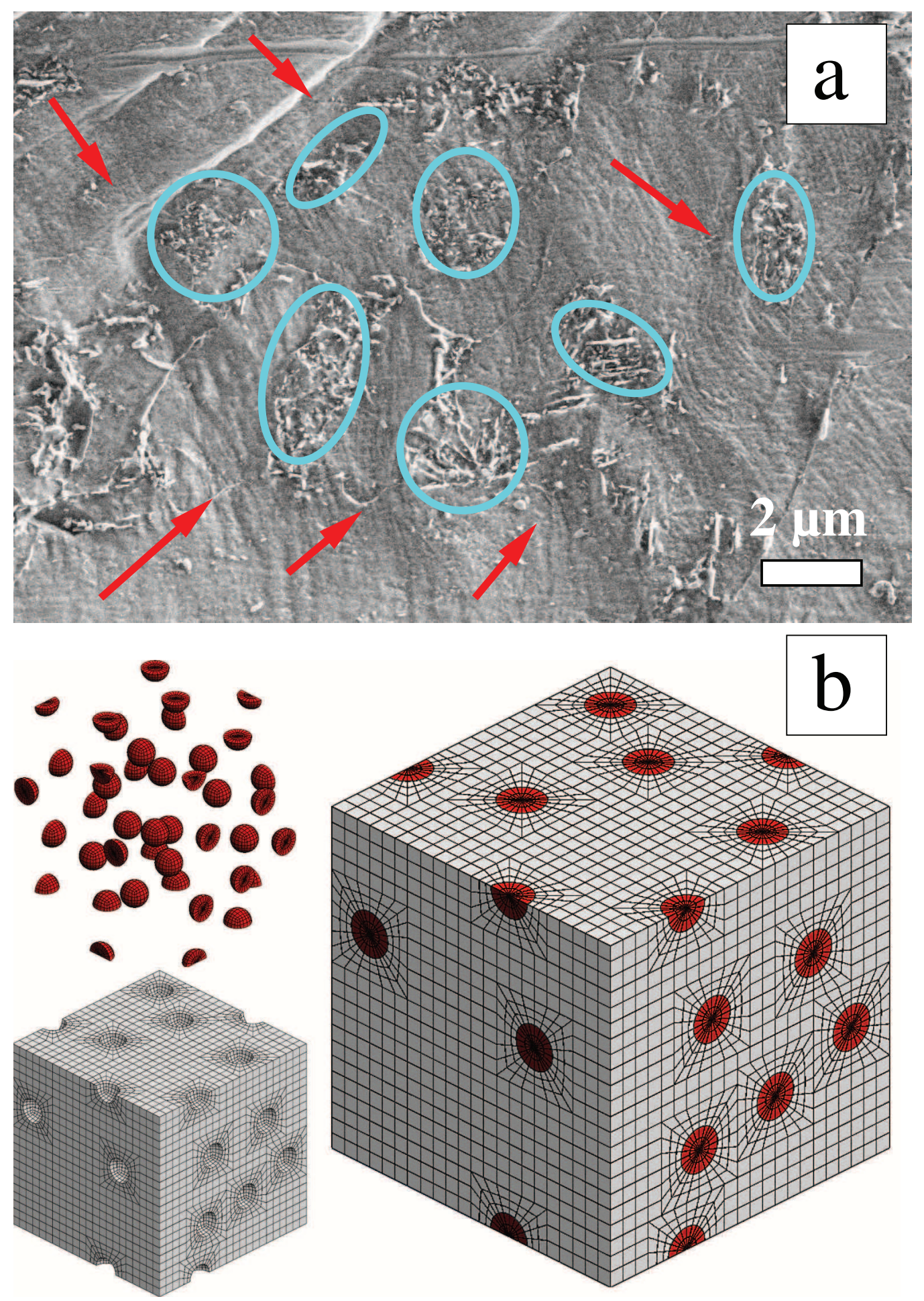

Fig. 4. Representation of carbide clusters : a) Plasticity flow lines circumventing clusters (indicated by arrows) and approximation of the clusters morphology, and b) representative mesh used for the calibration of $\delta$ and $D$ parameters. 
One should notice that random sampling of Euler angles to obtain an isotropic texture can not be done directly, but has to follow the following repartitions :

$$
\text { and }\left\{\begin{array}{l}
\varphi_{1} \text { follows an uniform repartition on }[0 ; 360] \\
\varphi_{2} \text { follows an uniform repartition on }[0 ; 360] \\
\cos (\Phi) \text { follows an uniform repartition on }[-1 ; 1]
\end{array}\right.
$$

Since lath packets are considered as morphologically isotropic, and have an approximate size of $20 \mu \mathrm{m}$, aggregate size used in the calibration procedure an be estimated approximately to $200 \times 200 \times 200 \mu \mathrm{m}^{3}$.

\subsection{Calibration procedure}

The calibration procedure of the modelling will be an iterative process since multiple scale computations have to be done. One can find a detailed explanation about the calibration procedure in [2]. The main aspects of calibration are the following : (i) Ferrite plasticity needs only 3 parameters to be identified, and they will be chosen as temperature dependent, and (ii) $\delta$ and $D$ parameters of the $\beta$ model were not found sensitive to ferrite behaviour change in the considered temperature range so that they will not be temperature dependent.

Calibrated parameter are presented in table 1.

\begin{tabular}{|c|c|}
$\begin{array}{r}\text { calibrated parameters } \\
(\beta \text { model })\end{array}$ \\
\hline$D$ & $\delta$ \\
\hline 900 & 0,4 \\
\hline
\end{tabular}

\begin{tabular}{|c|c|c|c|}
\hline \multicolumn{4}{|c|}{$\begin{array}{c}\text { calibrated parameters } \\
\text { (ferrite plasticity) }\end{array}$} \\
\hline $\mathbf{T}$ & $Q$ & $b$ & $\tau_{c 0}$ \\
\hline$-60^{\circ} \mathrm{C}$ & 3 & 90 & 204 \\
\hline$-90^{\circ} \mathrm{C}$ & 3,7 & 85 & 213 \\
\hline$-120^{\circ} \mathrm{C}$ & 4,1 & 60 & 231 \\
\hline$-150^{\circ} \mathrm{C}$ & 4,7 & 55 & 248 \\
\hline
\end{tabular}

Table 1

Result of the calibration procedure. 


\subsection{Results and experimental validation}

\subsubsection{Temperature dependence of the behaviour}

Results of calibration process in terms of simulated macroscopic response of the aggregate at different temperature can be found in figure 5 .

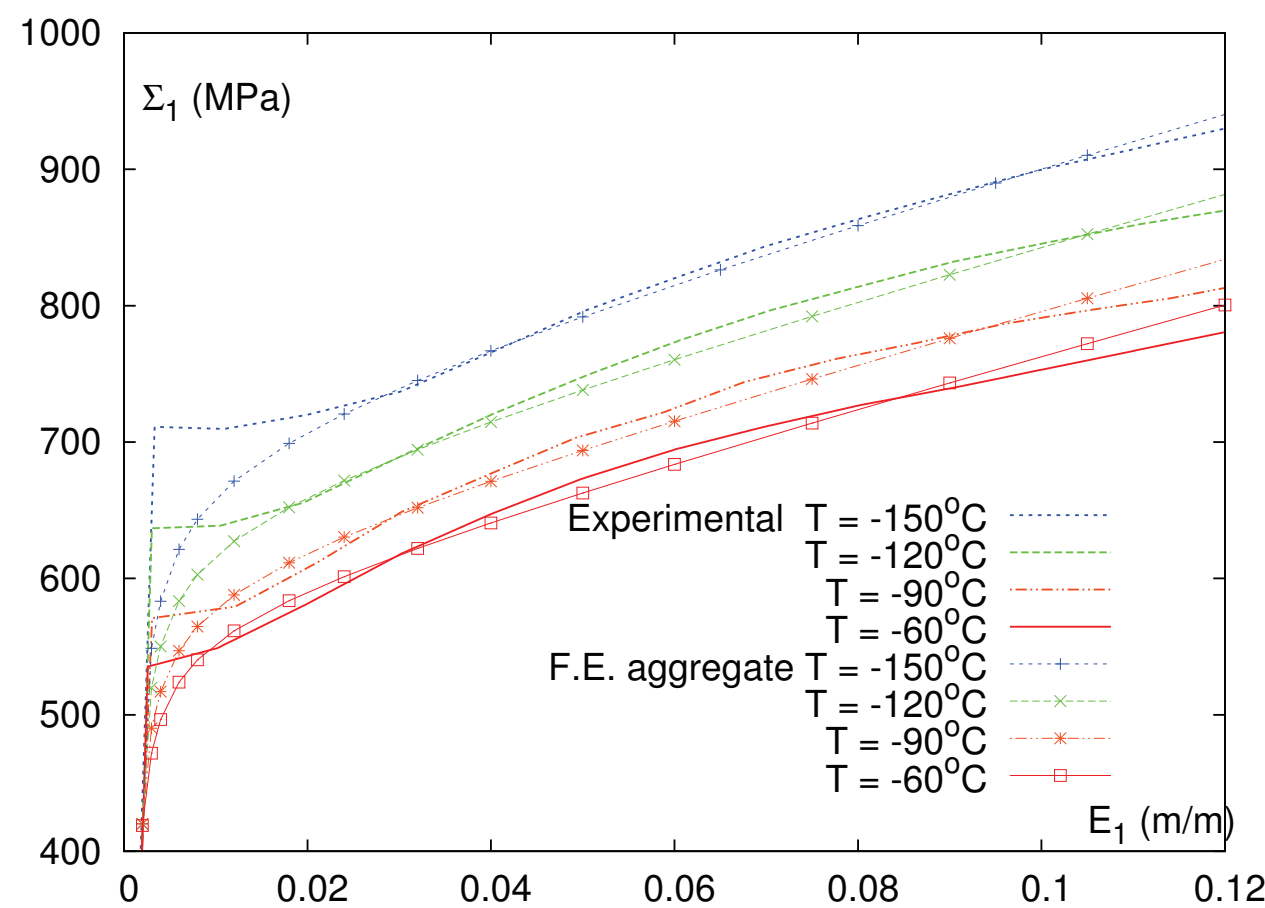

Fig. 5. Global simulated macroscopic response for different temperatures, compared with experimental results.

Experimental tensile tests results show a sudden onset of yielding, followed by an apparently quasi-plastic plateau. In fact, lots of instabilities also appear during this plateau which are due to plastic phenomena (Lüders bands). This phenomenon, which is increased with lower testing temperatures, has been removed from the experimental tensile curves. This is a classical problem for the definition of the beginning of the tensile curve with mechanical formulations used in this work: they cannot report neither the instabilities neither the quasi-static plateau. Nevertheless, it does not prevent a good agreement between experiments and simulation for strain levels relevant for fracture simulation. It should be emphasized that this good agreement at the macroscopic scale is thought by authors to be the result of modelling choices, since only 5 parameters were calibrated during the whole process. This can be considered as fully satisfying only if the process allows a good description of ferrite local stress states, which will be checked in sub-section 3.3.3. 


\subsubsection{Composite effect description}

The $\beta$ model parameters have been calibrated at $-150^{\circ} \mathrm{C}$ for one ferrite orientation during tensile straining on the representative morphology presented in figure 4. It is worth noting that validity of the calibrated parameters for different loadings (pure shear, and bi-axial straining) have been checked. Results indicated an overall good agreement between composite FE modellings and $\beta$ model and an excellent agreement about ferrite stress and strain state description [2].

One can find on figure 6.a the calibration results, represented by the comparison between $\beta$ model and FE composite model per phase stress repartition. This calibration allowed to reproduce quite well the average stress repartition in the ferritic phase. Figure 6.b presents the average repartition of stress between carbide clusters phase and ferrite phase in the bainite aggregate once the calibrated $\beta$ model is introduced as behaviour law at each IP. The difference in stress between the macroscopic stress level and the ferrite stress is comparable with the one experimentally determined by X-Ray diffraction thanks to the $\sin ^{2} \psi$ method. The experimental methodology is fully detailed in [25] and results are indicated in figure 6.c. It is worth noting that stress determination in ferrite by $\sin ^{2} \psi$ method implies an incertitude which can reach $\pm 20 \mathrm{MPa}$ at the end of tensile test.

Stress in carbide clusters is not fully represented in figures, because of their stress scales. It can reach values as high as $4900 \mathrm{MPa}$ in both FE composite model and $\beta$ model. This was not found critical: fracture modelling will mainly rely on ferrite stress state description.

\subsubsection{Polycrystalline effect}

Figure 7 represents the stress repartition inside the aggregate during tensile straining at $-150^{\circ} \mathrm{C}$. Some average lath packets behaviour is highlighted in figure 7.a to emphasize polycrystalline related heterogeneities. Simulated stress in ferrite matrix is also reported for these packets in this figure. One can notice that the difference in loading between ferrite and bainite (represented by red dots for a macroscopic strain of $12 \%$ in the aggregate) is related to the average behaviour of packets. Those that are submitted to the higher stress for a given macroscopic strain $(1,2)$ are also those that undergo less deformation (i.e. less plastic deformation because of a stronger hardening), so that difference in stress between bainite and ferrite is also lower. This means that the joint effect of polycrystalline and composite hardening in ferrite have to be taken into account to capture stress repartition in ferrite correctly.

Figures 7.c and 7.d present the additional second order polycrystalline effect captured by FE aggregate computations. The mechanical fields inside ferritic 

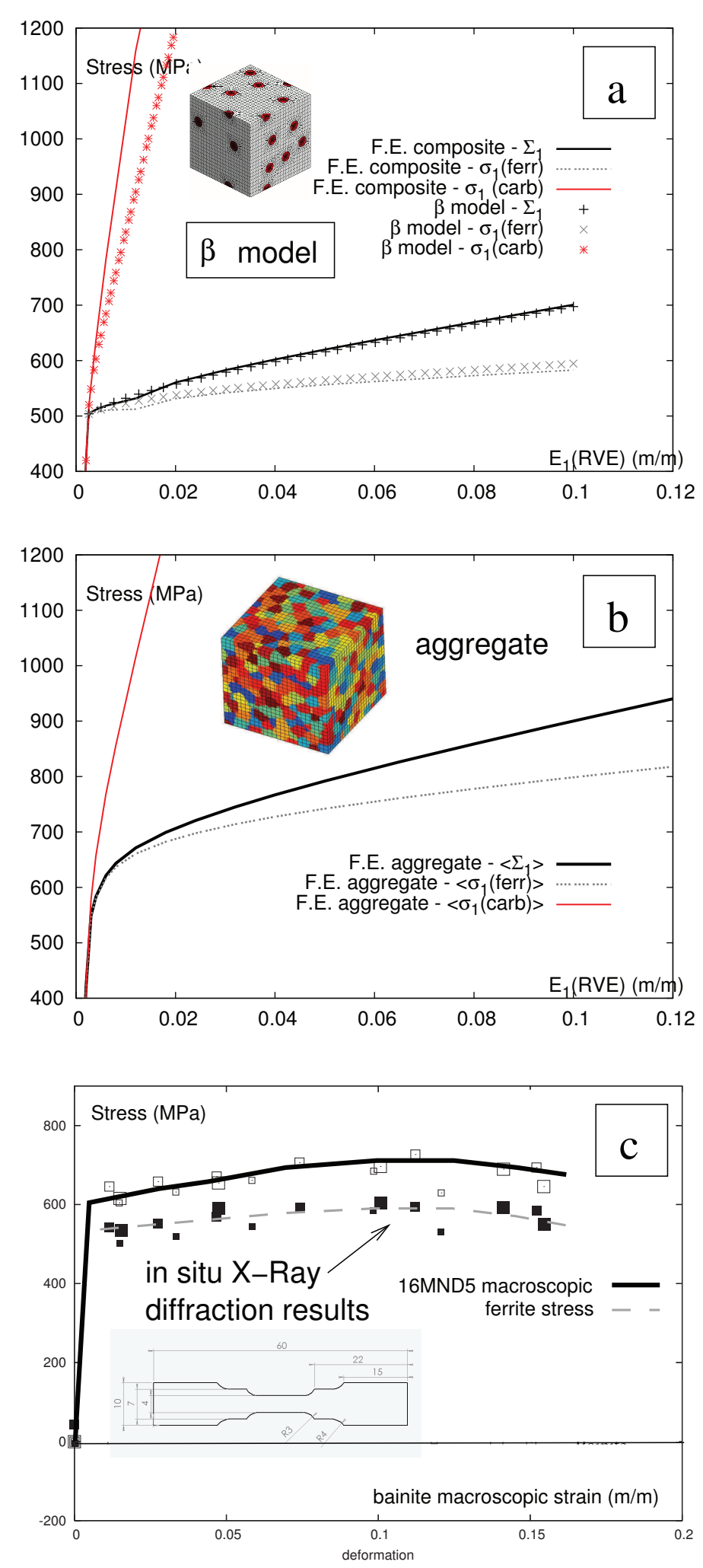

Fig. 6. Stress repartition between phases during tensile straining at $-150^{\circ} \mathrm{C}$ : a) for identified $\beta$ model and in average in the associated representative FE computation $\left(\varphi_{1}=18^{\circ}, \varphi_{2}=12^{\circ}, \Phi=82^{\circ}\right)$, b) In average in the aggregate. c) Stress difference between bainite and ferrite (incertitude for ferrite stress determination : $\pm 20 \mathrm{MPa}$ ) obtained experimentally during in-situ tegsile tests at $-150^{\circ} \mathrm{C}[2]$. 


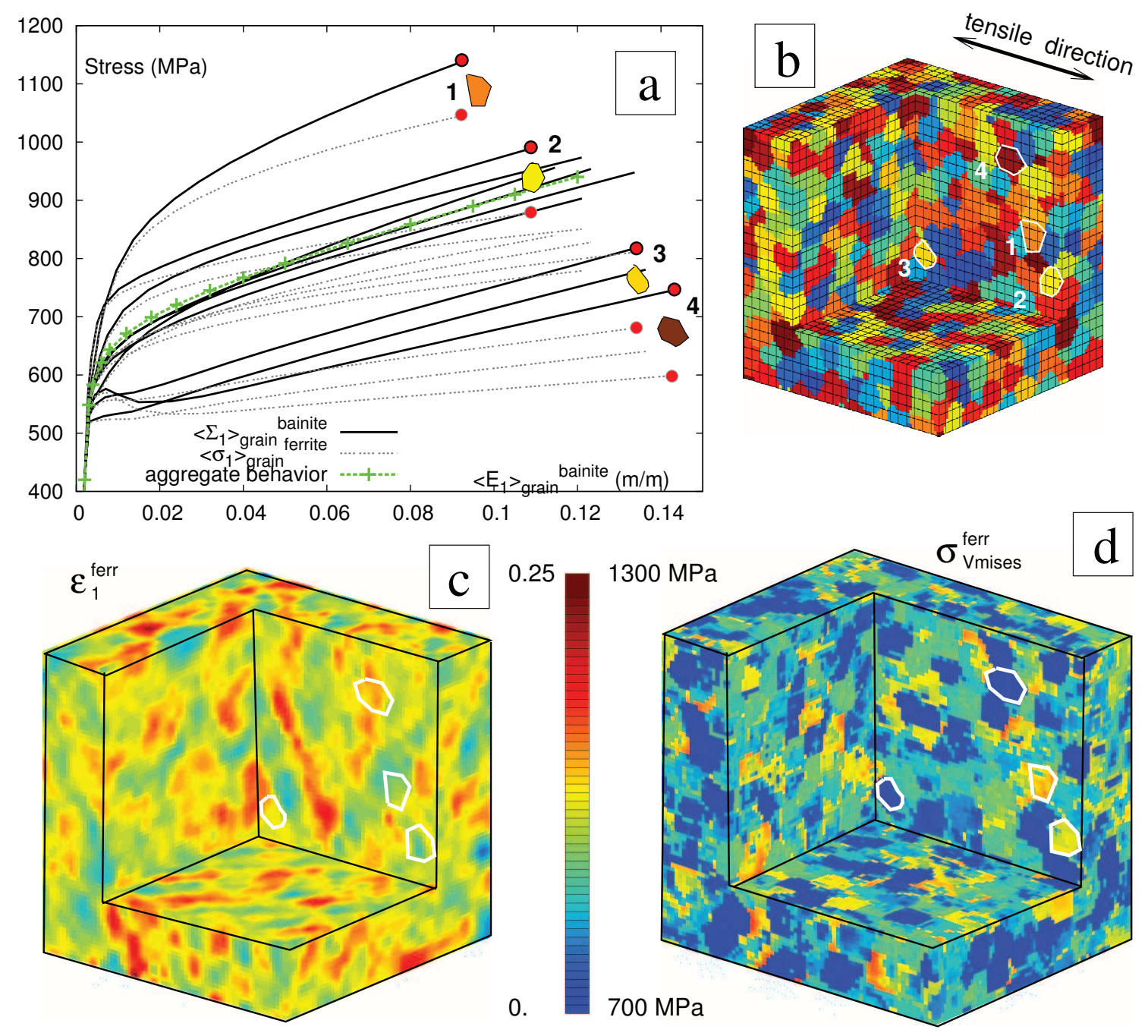

Fig. 7. Stress repartition inside the aggregate during tensile straining at $-150^{\circ} \mathrm{C}$. a) Illustration of some individual ferritic packets average behaviours, and of the associated average ferrite stress evolution. b) Representation of the represented geometry : a cut has been performed so that inner effects can be observed. Four packets have been highlighted so that they can be observed in other figures. c) Repartition of strain in tensile direction in the ferritic phase. d) Repartition of the von Mises stress in the ferritic phase.

packets are not homogeneous. It was chosen to show these fields inside the aggregate and not only at the aggregate surface since boundary conditions are known to induce some disturbance at the surface [42]. Figure 7.c shows preferential plasticity path common for such computations and also experimentally observed at the lath packets scale during in situ tensile testing. Figure 7.c allows to see that higher stress states in ferrite at the IP scale are present in highly loaded packets near the misoriented boundaries, where biggest carbides are found [11]. This last modelling feature can be easily explained as plastic accommodation mainly occurs at grain boundaries. This is considered to be 
the main advantage of FE aggregate computations over MF modellings for a precise description of local micromechanical ferrite stress state.

Experimental validation of inter-granular stress repartition is offered in addition with X-ray diffraction stress determination by $\sin ^{2} \psi$ method. It was fully exposed in $[2,26]$. This experimental work highlighted undulations of $\sin ^{2} \psi$ curves used for stress determination in ferrite after unloading during interrupted tensile testing at $-150^{\circ} \mathrm{C}$. This can be attributed to a quite heterogeneous residual stress state inside the differently oriented packets of ferrite. A post-processing was developed to simulate X-ray diffraction measurements on surfaces of FE aggregates submitted to unloading after tensile straining until $10 \%$ deformation. Figure 8 summarizes this work: this quite good agreement validates the modelling at the inter-granular scale although it was calibrated only at the macroscopic behaviour scale.

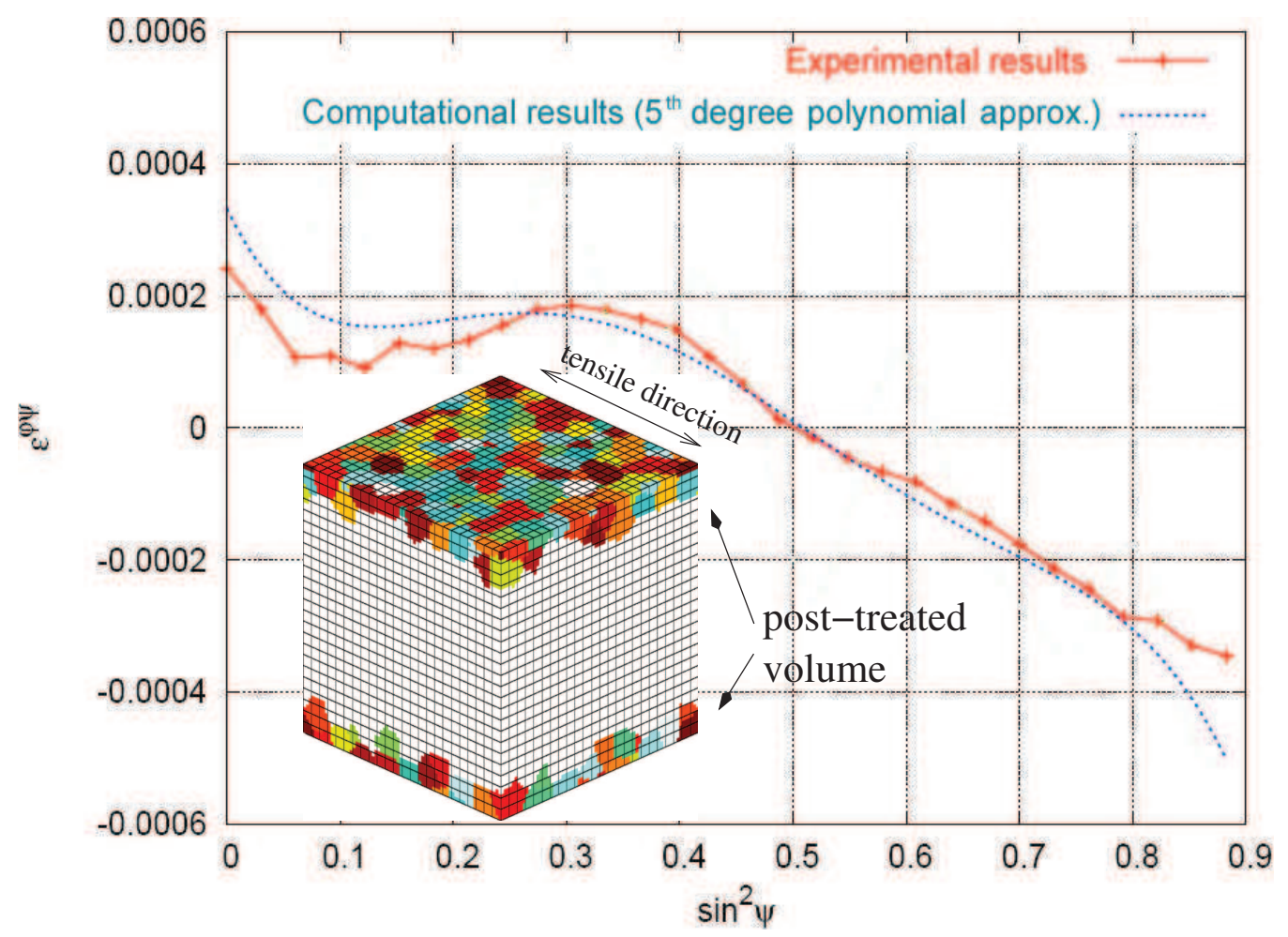

Fig. 8. Comparison between undulations of $\sin ^{2} \psi$ curves experimentally obtained and simulated on aggregates [26].

\subsubsection{Discussion about multiscale modelling and prospects}

The proposed multiscale approach that was setup in this part of the work is considered to have some advantages for further micromechanical fracture modelling work on bainitic steel. Although quite complex and allowing a detailed micromechanical description of ferrite micromechanical stress state, it 
does not require numerous adjustable parameters. Stress heterogeneities were also validated inside ferrite at the inter-granular scale, and between ferrite and bainite at the macroscopic scale without further calibration.

It is worth noting that recent research works, mainly conducted within the PERFECT project, improved and sometimes gave confirmation of the validity of some modelling choices on different specific aspects of stress heterogeneities description inside 16MND5 steel:

- Micromechanical ferrite behaviour description can be based on lower scale description. It is the case in Libert's work [43], its approach taking dislocations densities on gliding systems $n$ as the lower scale internal variable ( a contrario, the present work uses gliding on a slip system). This allows to take into account easily the change of dislocations interactions mechanisms with temperature, which leads to a finest description of hardening mechanisms for different temperatures.

- Recent work about dislocation dynamics [44] gave precise information about interactions between gliding systems, making more precise calibration of the interaction between systems in micromechanical modellings possible (i.e. the $h^{g h}$ matrix of this work could be based on such simulations).

- An isotropic texture was used in the present work, on both morphological and crystallographic point of views. However, it is well known that lath packets relative orientation follow local misorientation rules due to ferrite variants growing during austenite to ferrite transformation [45]. Recent efforts [46] permitted to enlarge greatly simulated domains and thus to improve the precision of their morphological description: lath packet morphology and local misorientations between lath packets were taken into account in large-scale computations, and several studies of their influence on the stress repartition inside aggregates were made. Results indicate that these relative crystallographic orientations influence the stress repartition whereas the packet morphology (in opposition to the grain morphology) does not play such an important role.

- Experimental work conducted in this work relies on inter-granular stress heterogeneities description, but new techniques arises, like Kossel microdiffraction that could allow to investigate intra-granular stresses at the micron scale [47]. 


\section{Modelling of brittle fracture}

\subsection{Critical mechanisms for cleavage fracture}

In order to establish an efficient cleavage criterion at the microscopic scale, one has to determine which one(s) of the three steps (initiation, propagation, crossing of microstructural barriers) have to be considered as the critical one(s), and what mechanism(s) can be considered to govern it(them).

\subsubsection{Cleavage initiation}

Different micromechanisms have been proposed in the past for initiation of a microcrack in low-alloyed steels. Zener [1] and Stroh [48] were the first to associate cleavage and dislocations pile-ups at interfaces (grain-grain, particlematrix). Cottrell [49] proposed a similar mechanism, where interaction between dislocations can create initiation, and Friedel [50] observed initiation triggered by twins. Carbide cracking initiation at grain boundaries was proposed by Smith [51]. Whatever mechanism is considered, one can notice that it is always initiated by plastic activity.

Different fractographic studies where conducted on the studied material. Carbides are generally shown to be the initiation sites in such steels $[52,53]$, but they can't always be clearly identified at the initiation of "cleavage rivers" [54]. On the other hand, other second phase hard particles can be found [55]. Ortner [56] reports that if a second phase particle can not always be found at the supposed initiation site, those particles may have been expelled. Chemical analysis sometimes confirms this hypothesis by showing residual traces of particle presence.

Carbide cracking will thus be considered as the initiation mechanism. Since every work reports influence of plastic deformation, a triggering value will be considered for initiation.

\subsubsection{Cleavage propagation to contiguous matrix}

In the work of Im et al. [53], it is also shown that cracks appearing for smallest carbides belonging to clusters in bulk material were not propagated but blunted by ferrite plasticity.

This last point justifies that the distinction between biggest carbides and small ones has to be made in this work. A Griffith-like [57] propagation criterion $\sigma_{p}$ will thus be used for propagation of a "penny-shaped" defect of radius $r_{d}$, 
based on the initial defect size (i.e. carbide size) in ferrite :

$$
\sigma_{p}=\sqrt{\frac{\pi E \gamma_{R}}{2\left(1-\nu^{2}\right) r_{d}}},
$$

where $\gamma_{R}$ represents the Effective Surface Energy (ESE) which is the surface energy of the lattice at 0K plus the energy involved in other dissipative mechanisms, depending on the phenomenon (i.e. blunting or dislocation emission at the crack tip for crack propagation, or getting over misoriented boundaries for microstructural barriers crossing).

This criterion is widely used for brittle propagation modelling. It will explicitly prevent the propagation of smallest cracked carbides. On the other hand, it is worth noting that if the smaller carbides clusters can thus appear completely uncorrelated from fracture, the way they are taken into account in the behaviour modelling (composite effect) will influence the microscopic stress state in ferrite, and thus propagation.

\subsubsection{Microstructural barriers crossing}

Once a crack has been propagated to the surrounding ferrite inside a lath packet, it has to cross packet boundaries, which can be seen as a grain boundary. This event was not considered as a critical one in this work for two reasons.

Few experimental proofs of such microscopical scale cleavage arrest exists in 16MND5 steel. When they do [58], it is not clear whether they were propagated before principal cleavage and then stopped. Another plausible hypothesis being that they resulted from the high stresses concentrations in the vicinity of the main cleavage crack tip.

From a theoretical and modelling point of view, barrier crossing could be seen as another Griffith like mechanism. In this case it would be easy to consider two propagation stress:

- The "propagation" stress would be associated to ferrite lattice ESE, and carbide sizes.

- The "barrier crossing" stress would be associated to misoriented lattice interface ESE crossing, and to lath packets sizes.

Lots of estimations can be found for ESE of propagation inside bainitic steels and ferrite $[4,59,53,56]$, but also for ferrite lattice misorientation crossing $[59,60]$. The associated carbides sizes and lath packets inside 16MND5 microstructure have been thoroughly characterized, allowing to represent plausible critical stresses for both mechanisms in figure 9 . 


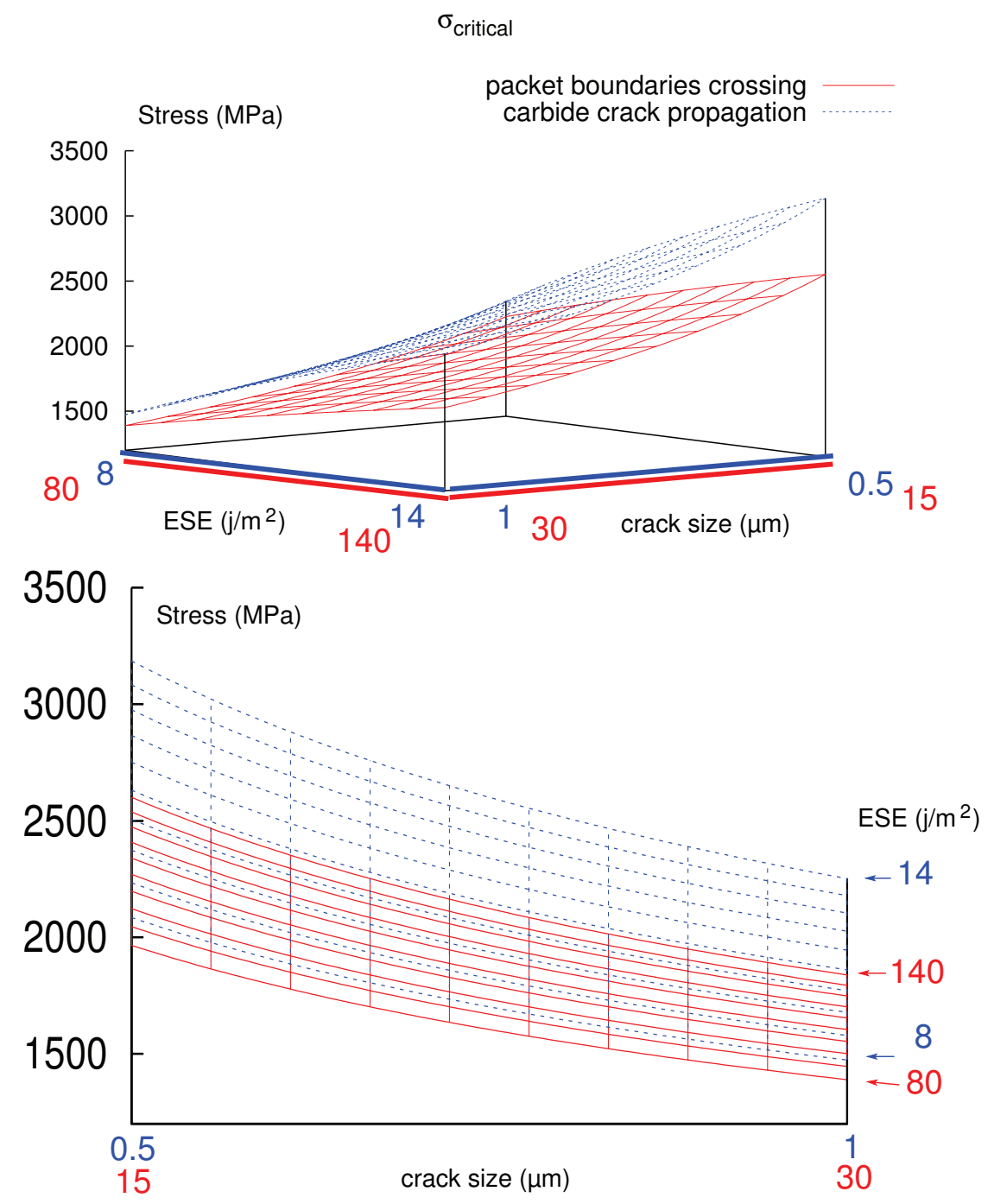

Fig. 9. 3D surfaces representing the possible critical stresses for propagating a penny-shape crack in case of lath packet boundaries crossing and of carbide crack propagation to contiguous ferritic lattice. Two views are presented for readability purpose.

The figure indicates that stress required for carbide crack propagation is globally higher than the one required to cross barriers. However, this figure shows that the stress domains are quite close one to each other. The domains where represented in the same 3D plot (with different scales) for comparison purpose.

It is worth noticing that ESE estimations, especially for boundary crossing, are to be considered with high cautiousness. In fact those values are usually deduced from an inverse calibration process based on a Griffith criterion [60]. This means that they are related to identification procedure that rely on a macroscopic stress state estimation, and an a priori mechanism estimation, 
although it is not always clear whether one or the other mechanisms was really identified as responsible for fracture. So that they may not be relevant for micromechanical considerations.

In case of finer bainitic microstructure, like those obtained after welding of low-alloyed steels, average laths packet size can become quite smaller than for 16MND5 steel, and one may have to take microstructural barriers crossing into account to correctly predict cleavage [61]. In the case of 16MND5 steel, it is the propagation step that will be considered as critical.

\subsection{Cleavage modelling assumptions and methodology}

The hypothesis about cleavage used in this work are based on Beremin's workgroup assumptions, and adapted to previously discussed critical mechanisms. They give a framework for a local approach to fracture applied at the micromechanical scale to 16MND5 steel. In the following, those hypothesis are enunciated in relation to equations used to apply a deterministic fracture criterion on aggregate computations.

\subsubsection{Initiation}

Carbides are the initiation sites for micro-cracks. The initiation will be triggered by a criterion on plastic equivalent strain at each IP $i$ :

$$
\varepsilon_{p, i}^{e q u} \geq \varepsilon_{p}^{m i n i}
$$

\subsubsection{Effective crystallographic cleavage stress}

Once a defect is initiated, propagation in the surrounding ferritic matrix is a "Griffith-like" process. Cleavage in iron BCC lattice occurs mainly by separation of crystallographic planes of the $<001>$ family, three local stresses acting on cleavage planes will thus be computed according to local lattice orientation (Euler angles). Only the maximum value of the three computed can be considered as the effective one for cleavage:

$$
\sigma_{c, i}^{M a x}=\max \left(\underline{n}^{p} \cdot \sigma_{f e r} \cdot \underline{n}^{p}\right)_{i} \text { with } p \in[100 ; 010 ; 001] .
$$

This allow to compute a value of the effective crystallographic cleavage stress $\sigma_{c, i}^{M a x}$ at each IP $i$ of the aggregate. 


\subsubsection{Triaxial stress state loading on the aggregate}

Previous work highlighted that cleavage initiation of steels involves high local stress values, that cannot be accessed using uni-axial loading on RVE [24]. Thus a triaxial stress state was applied to the computed aggregate. This was obtained by increasing the hydrostatic part of the stress tensor $\mathbf{S}$. Loadings are thus controlled by macroscopic stresses tensor applied on the aggregate and stress triaxiality will be represented by $\chi$ :

$$
\chi=\frac{1+2 \alpha}{3(1-\alpha)} \quad \text { with } \quad S_{2}=S_{3}=\alpha S_{1}
$$

It is worth noticing that $\mathbf{S}$ diagonal components can also be taken as its principal values in this setting $\left(\Sigma_{I}=S_{1}\right)$, and that the stress triaxiality remains a constant during loading of the aggregate. This was considerate as quite important to separate mechanical effects (triaxiality) from thermal effect: during experiments on cracked or notched specimen at different temperatures, the change in material behaviour makes this separation difficult. This is to be considered as an advantage of the present approach: except with such numerical tools, it is quite difficult to consider loadings at a constant stress triaxiality.

Boundary conditions perturbations at the surface were cautiously avoided [2]. This was done by performing aggregate simulations on a larger volume than needed, and extracting only the central part of interest $V_{0}$. The volume $V_{0}$ was chosen consistent in size with the Beremin local approach EV generally used for bainitic steels $\left(50 \times 50 \times 50 \mu \mathrm{m}^{3}\right)$. The aggregate meshing used will be the one represented in figure 3. Figure 10 shows the repartition of maximum cleavage stress $\sigma_{c}^{\text {Max }}$ and of the equivalent plastic strain $\varepsilon_{p}^{\text {Mises }}$ in ferrite for the EV $V_{0}$, extracted from this aggregate which is loaded with a triaxial stress state.

\subsubsection{Carbide population and Griffith stress for propagation}

An approximated statistical repartition function based on experimental carbide size distribution (given in figure 11.a) will be considered in this work. It was provided by Ortner et al. [56] and was extracted from German Euro "A" steel study. This steel is similar to french 16MND5 material :

$$
p\left(r \geq r_{0}\right)=1-\exp \left(-\left(\frac{r_{0}}{3.6 \cdot 10^{-8}}\right)^{-2.7}\right),
$$

It is worth noting that experimental work provided in [62] gives a similar experimental carbide repartition for an A508 Cl.3 steel, although approximated statistical repartition differs [2]. Statistical repartition from Ortner comes with 

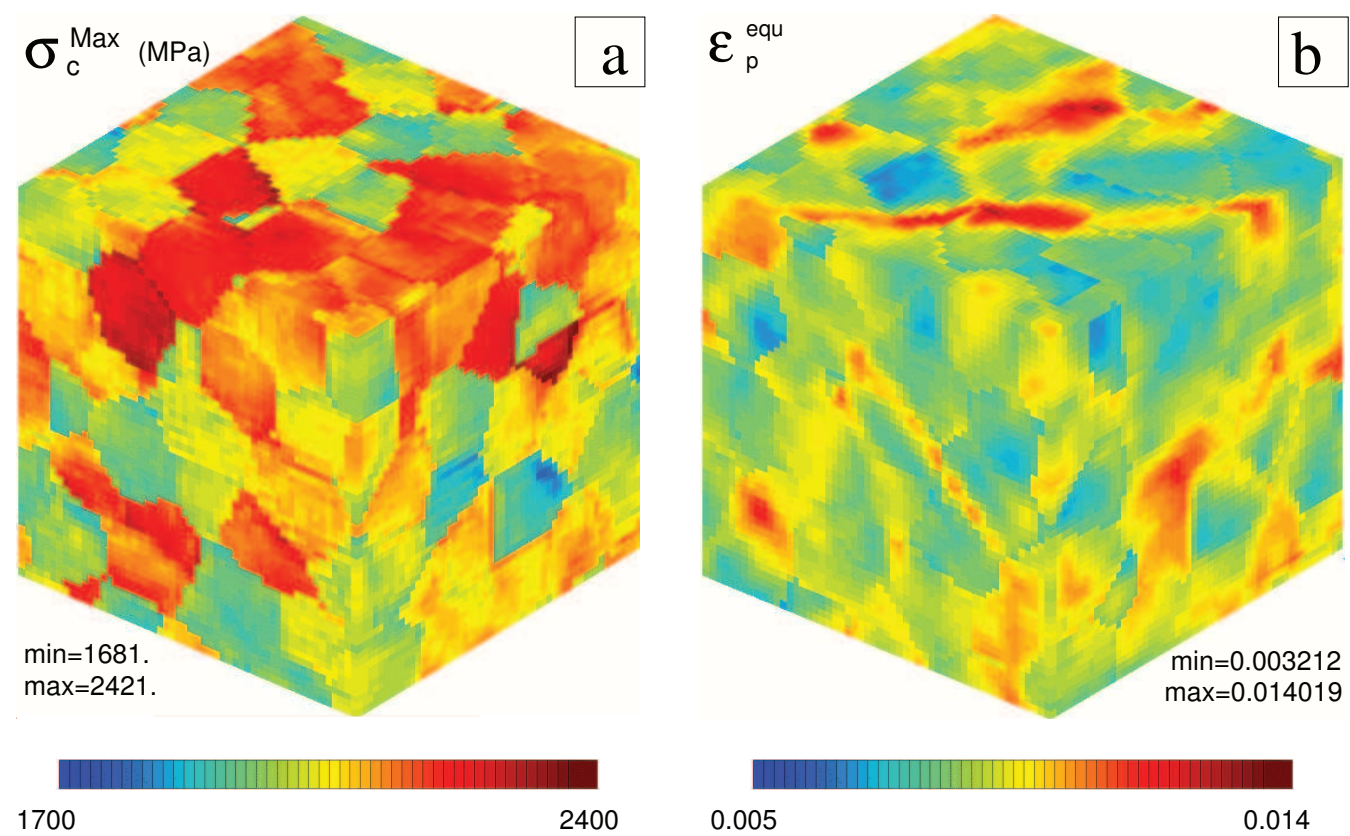

Fig. 10. Repartition of a) effective crystallographic cleavage stress and b) equivalent plastic strain in ferrite during triaxial loading on the aggregate. $\mathrm{T}=-150^{\circ} \mathrm{C}, \chi=3$, $\Sigma_{1}=2200 \mathrm{MPa}$

a precise carbide volume counting $\left(N=7.6 \pm 2.4 \cdot 10^{17} \mathrm{~m}^{-3}\right)$, which lacks in the other work.

As already mentioned, the carbide population is divided into a population of small carbides gathered in clusters that was taken into account during the behaviour modelling, but also into a population of bigger lonely carbides eligible for plastic crack initiation. Thus this global carbide size distribution can be directly used to reproduce random sampling of carbide sizes at each IP, the distinction between the two populations roles being a natural process.

Inside the EV the repartition can be used to attribute an average number of carbides $\bar{n}$ at each IP of 0.603 , knowing its size $V_{0}$, its number of IP $(18 \times 18 \times$ 18 elements with 27 IP),

A truncated Gaussian distribution of integers (average $\bar{n}$, with standard deviation $\omega_{n}=4$, truncated below 0 ) is used to attribute a carbide number $n_{i}$ at each integration point $i$. One can observe such a distribution in figure 11.b. Randomly attributed sizes $r_{n, i}$ are then attributed to these $n_{i}$ carbides using the repartition given in equation 21 .

The propagation stresses $\sigma_{n, i}^{p}$ are then deduced at IP $i$ according to the Griffith's criterion (equation 17) and the randomly attributed carbide size. The biggest carbide sizes obtained by random realization using equation 21 can reach non realistic values. It was thus chosen to introduce a threshold 


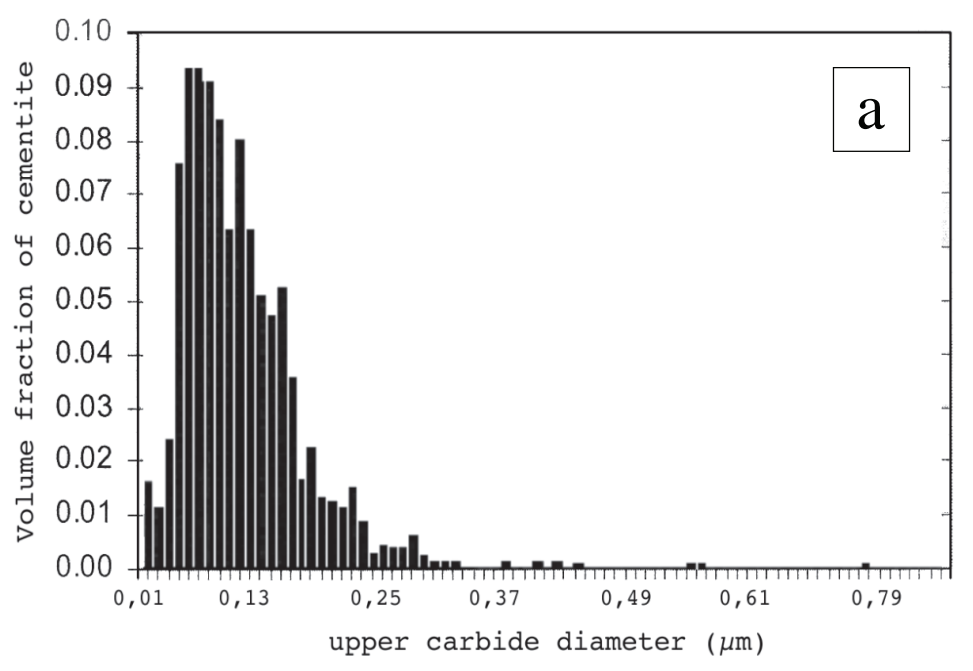

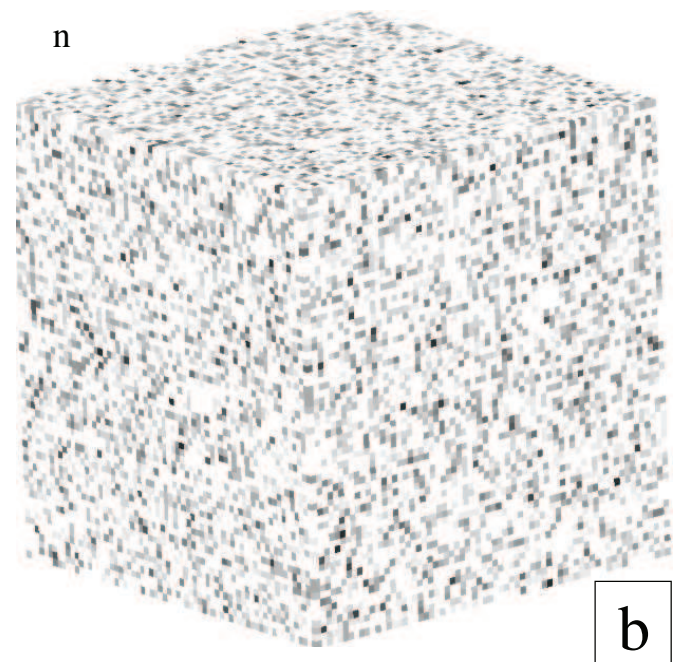

0

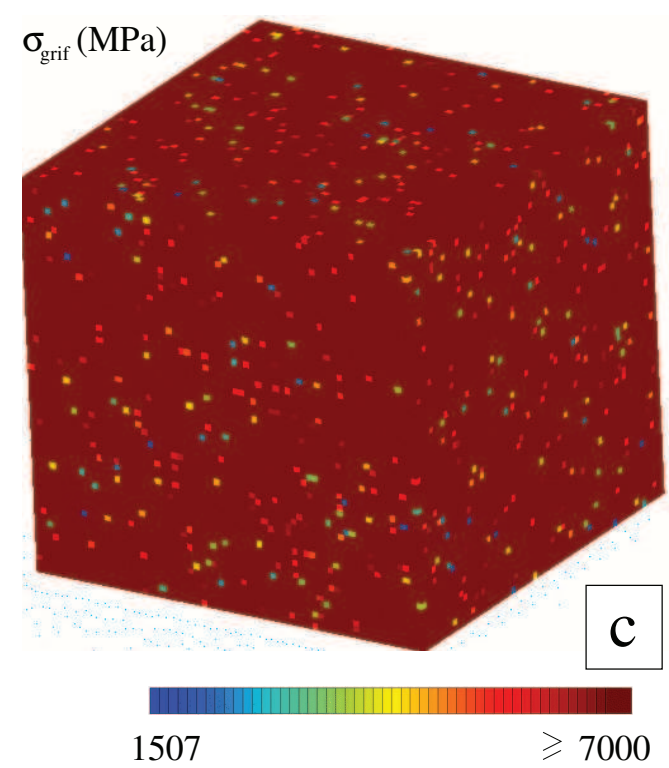

Fig. 11. a) Experimental repartition of carbides sizes represented with one deterministic realization based on equation 21. b) Repartition of carbides in EV and c) resulting Griffith stress repartition

to eliminates those values. This threshold value was chosen in accordance to the biggest carbides observed $\left(r_{\max }=1 \mu \mathrm{m}\right)$ in the material $[63,11]$. The propagation stress $\sigma_{n, i}^{p}$ will thus be chosen as an arbitrary unreachable "nonpropagation" stress value $\sigma^{N P}$ for carbides sizes upon $r_{\max }$, with respect to this hypothesis :

$$
\sigma_{n, i}^{p}=\left\{\begin{array}{ccc}
\sqrt{\frac{\pi E \gamma_{p r o p}}{2\left(1-\nu^{2}\right) r_{n, i}}} & \text { if } & r_{n, i} \leq r_{\max } \\
\sigma^{N P} & \text { if } & r_{n, i}>r_{\max }
\end{array}\right.
$$


$\gamma_{\text {prop }}$ represents the ESE of the ferrite lattice. The Griffith critical stress $\sigma^{\text {grif }}$ at an IP $i$ will be the smallest value of $\sigma_{n, i}^{p}$, and will be taken equal to $\sigma^{N P}$ when no carbide was attributed, as described in the following equation :

$$
\sigma_{i}^{g r i f}=\left\{\begin{array}{ccc}
\min \left(\sigma_{n, i}^{p}\right) & \text { if } & n_{i}>0 \\
\sigma^{N P} & \text { if } & n_{i}=0
\end{array}\right.
$$

Such a repartition of critical stress $\sigma_{i}^{\text {grif }}$ inside the RVE is represented in figure 11.c.

\subsection{Fracture event, and link with local approach}

Microstructural barriers are not considered as relevant during 16MND5 brittle fracture, so that the propagation of a previously initiated micro-crack is a "weakest link type event". This means that global fracture of the EV is considered to happen once both the initiation and the propagation steps are satisfied at any IP $i$ :

$$
\text { and }\left\{\begin{array}{l}
\varepsilon_{p, i}^{e q u} \geq \varepsilon_{p}^{\operatorname{mini}} \\
\sigma_{c, i}^{\max } \geq \sigma_{i}^{\text {grif }}
\end{array}\right.
$$

Fulfillment of the propagation step 24 can be seen as the moment where, at any IP, plastic activity reaches the threshold value, and one of the value illustrated in figure 10.a reaches the associated value in figure 11.c. A linear interpolation was used to compute the time and the first principal stress at failure between simulated time steps.

The random realization of carbides repartition can then be computed $X$ times, and for each computation $x$, a different first principal stress $\Sigma_{I}$ to fracture is computed: heterogeneous stress repartition in the aggregate remains the same each time, but the difference in carbide sampling induces a different place and time for equation 24 to be fulfilled. This would not have been the case without the micromechanical stress heterogeneities representation.

\subsection{Relation with Beremin's approach}

This leads to $X$ values of first principal stress to fracture. They were sorted in increasing order (i.e. $x=1$ for the smallest $\Sigma_{I}$ value). A cumulative probability 
of fracture can then be plotted as a function of $\Sigma_{I}$ stress to fracture using the estimator $P_{f}=(x-0.5) / X$.

A first computation was made to evaluate the process. It was chosen to attribute "usual" values found about pure cleavage fracture in literature to the different parameters [2]. The shapes of the curve, presented in figure 12, recalls the Beremin's failure cumulative probability evolution shape, and fits quite well with the Weibull equation. Gnuplot free software was eventually used to calibrate $m$ and $\sigma_{u}$ parameters to fit equation 1 with the plotted cumulative probability, assuming a Weibull distribution shape.

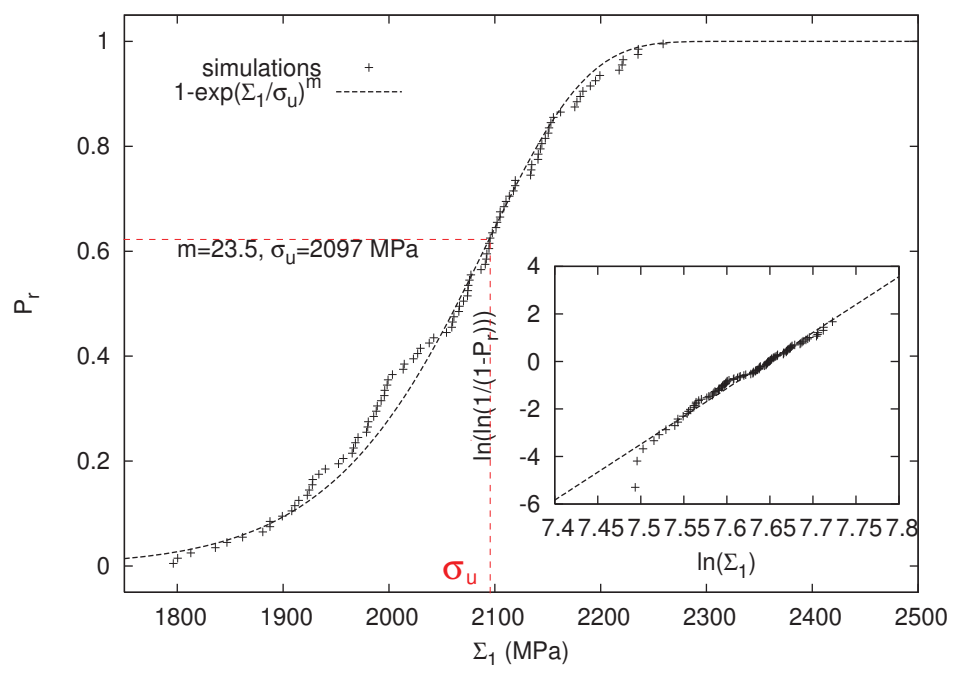

Fig. 12. Example of cumulative fracture probability of the EV obtained for : $\chi=2$, $\mathrm{T}=-150^{\circ} \mathrm{C}, r_{c}^{\max }=1 \mu \mathrm{m}, \gamma_{\text {prop }}=12 \mathrm{~J} \cdot \mathrm{m}^{-2}, \varepsilon_{p}^{\operatorname{mini}}=10^{-5}$. Result are fitted with equation 1.

This cumulative probability was obtained with the same loading $\mathbf{S}$ on the different EV. One can remark that plasticity was triggered for every deterministic fracture realization. Therefore, the obtained cumulative probability of fracture is equivalent to the cumulative probability that one would obtain by considering a macroscopic structure composed of $X \mathrm{EV}$ identical to this one, assuming that (i) they are globally loaded with $\underset{\sim}{\mathbf{S}}$ stress state, and (ii) their carbide population can be described by equation 21 . Thus, the whole process is similar to the use of Beremin's approach considering this virtual macroscopic volume composed of $X \mathrm{EV}$, with $\sigma_{w}=\Sigma_{I}$.

It is one of the remarkable originality of this whole approach: considering carbides realistic repartition has already been done in other works $[62,64]$, but one also has to consider the stress spatial repartition, not only at the macroscopic structure scale (structural, crack or notch effect), but also at the micromechanical scale inside an EV. This allowed to point out the fact that stress triaxiality on the EV influences the stress heterogeneities so that the 
probability of fracture of the EV is modified [26]. Next section is an attempt to apply a similar scheme to the temperature effect.

\section{Temperature dependence of Weibull parameters}

\subsection{Necessity of a temperature dependent value of $\gamma_{\text {prop }}$}

$X=100$ was found to be a good compromise between computation time and precise identification of $m$ and $\sigma_{u}$ parameters.

A stress triaxiality $\chi=2$ will be used for the study of temperature dependence, since it is a value commonly observed at the initiation site of cleavage at low temperatures [54]. It is worth noting that a study of the dependence of Weibull parameters to initiation criterion $\varepsilon_{p}^{\operatorname{mini}}$ was conducted for two triaxialities $(\chi=1.75$ and $\chi=3)$, and no dependence was found for $\varepsilon_{p}^{\min i} \in\left[10^{-6}: 10^{-3}\right]$, at $-150^{\circ} \mathrm{C}[2]$. At higher temperature, plastic activity at the time of failure will increase, so that initiation criterion won't be considered as critical in this work. Anyway, high local stress levels needed for propagation step only happens at IP where lots of plasticity occurred, so that initiation criterion is always fulfilled.

Values of $\gamma_{\text {prop }}$ in a large range are reported in literature. As a first approach, a value of $10 \mathrm{~J} . \mathrm{m}^{-} 2$ will be used, since it is an intermediary value between recently proposed values for 16 MND5 and similar steels $[11,53,56] . r_{c}^{\max }$ and $\varepsilon_{p}^{m i n i}$ will keep the same value than in the previous section. Figure 13 shows the results of the approach when considering these values.

This result does not indicate any important dependence of Weibull parameters with temperature but does not appear satisfactory considering efforts done in other works to take this dependence into account. In order to interpret this result at the micro-scale, one should examine figure 14 , which indicates the statistical repartition of the effective cleavage stress $\sigma_{c}^{\text {Max }}$ inside the EV for a macroscopic loading (i.e. $\Sigma_{I}$ value) comparable to the determined $\sigma_{u}$ value for each temperature, which, in our case corresponds to the mean value to fracture.

This explains clearly this previous result: although material behaviour evolves with temperature, the micromechanical stress repartition that will drive cleavage propagation does not change drastically with temperature for a constant value of ESE $\gamma_{\text {prop }}$. This may be explained in our case by the fact that the identified behaviour reveals hardening slopes quite similar for those different temperatures. Moreover, the considered constant value for $\gamma_{\text {prop }}$ implies that 

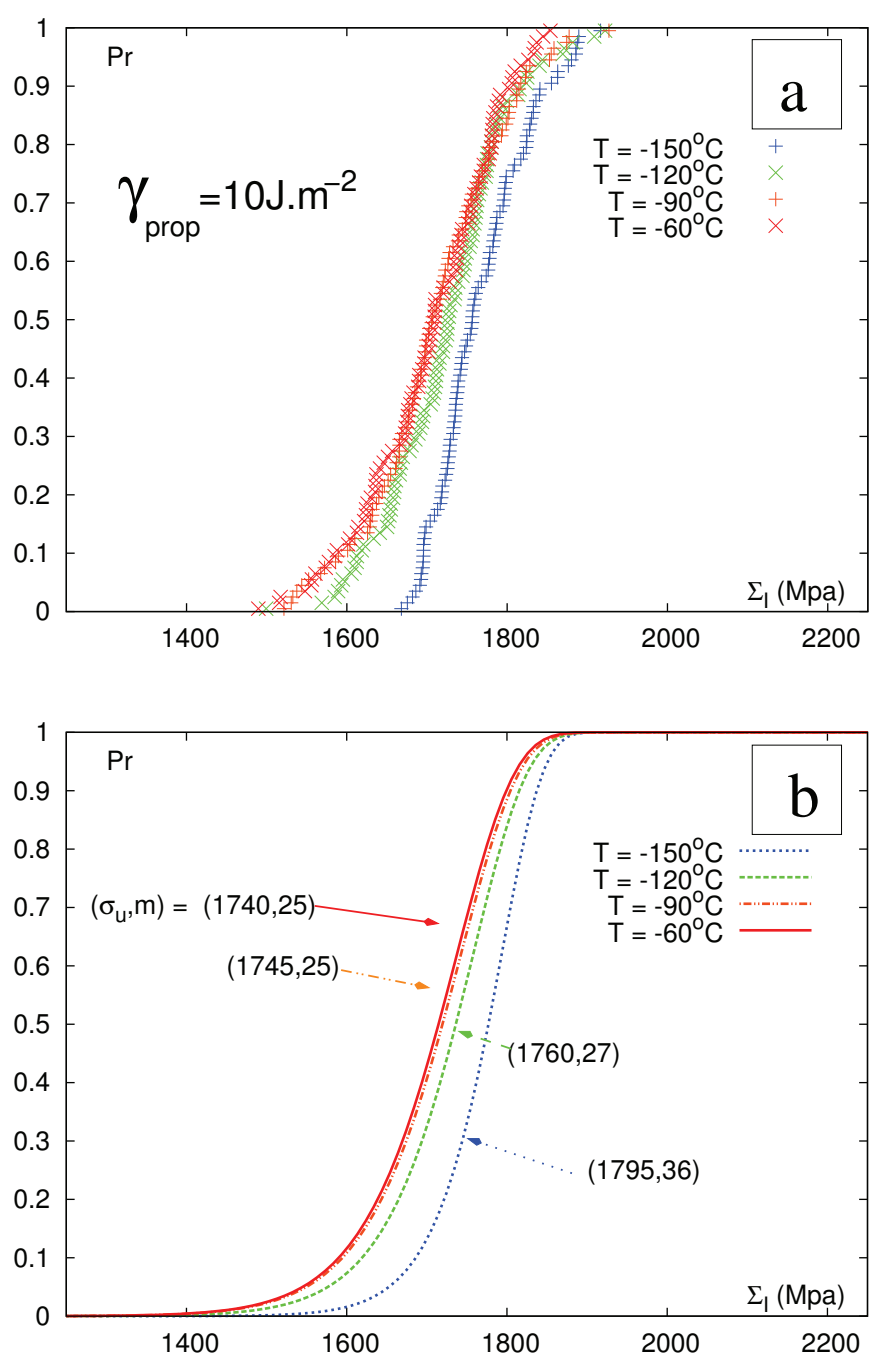

Fig. 13. Results of simulation of cumulative fracture of the EV for a constant value of $\gamma_{\text {prop }}$. a) Computation results and b) fitted Weibull parameters.

propagation stress $\sigma^{\text {grif }}$ inside the EV remains statistically identical at the different considered temperatures.

\subsection{Application of the Wallin hypothesis on $\gamma_{\text {prop }}$}

In order to capture the temperature dependence of the Weibull parameters, $\gamma_{\text {prop }}$ will be considered as temperature dependent, all the other parameters and hypothesis remaining the same as in the previous section. Wallin et al. proposed a phenomenological temperature dependence of the ESE related to the Peierls-Nabarro force :

$$
\gamma_{\text {prop }}(T)=\gamma_{\text {prop }}^{(O K)}+A \cdot e^{(B . T)} .
$$




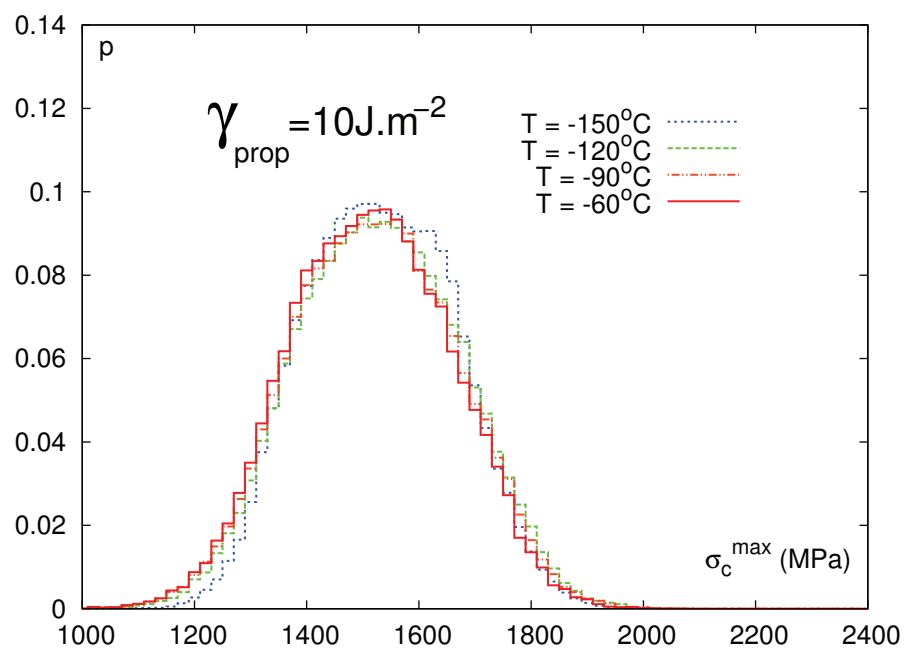

Fig. 14. Probability of the effective cleavage stress $\sigma_{c}^{M a x}$ inside the EV for value of $\Sigma_{I}$ comparable to the identified $\sigma_{u}(1750 \mathrm{MPa})$ for triaxial loading at different temperatures.

Wallin assumption about temperature dependence implies that the ESE increases with temperature. The calibrated values of Wallin's work are not consistent with values reported for 16MND5 steel, since the studied steel grade was not the same. A calibration will thus be done, but the choice was made to rely on existing values of ESE in literature rather than doing an inverse calibration on fracture experiments, to preserve the deductive aspect of the present analysis.

$\gamma_{\text {prop }}^{(O K)}$, which represents the surface energy at $0 \mathrm{~K}$ has been widely estimated by different approaches, a recent estimation of $2.15 \mathrm{~J}^{-\mathrm{m}^{-2}}$ [65] will be used. Values of ESE reported in literature in the studied temperature range ([$\left.150^{\circ} \mathrm{C}:-60^{\circ} \mathrm{C}\right]$ ) where found between $7 \mathrm{~J}^{-2}{ }^{-2}$ [56] and $12.4 \mathrm{~J} . \mathrm{m}^{-2}$ [53]. These values will be chosen respectively as the ESE value for $-150^{\circ} \mathrm{C}$ and $-60^{\circ} \mathrm{C}$. Gnuplot fitting module was used to fit $A$ and $B$ parameters according to these hypothesis. This leads to the following equation :

$$
\gamma_{\text {prop }}(T)=2.15+1.53 . e^{(0.009 .(T+273.15))},
$$

T being expressed in degree Celsius. The calibrated $\gamma_{\text {prop }}(T)$ is presented in figure 15.a, with other values found in literature. Figure 15.b shows fracture computations made using this temperature dependence, and fitted Weibull parameters are presented in figure 15.c. One should remark that computation made for a temperature of $-60^{\circ} \mathrm{C}$ is incomplete : aggregate computations were interrupted because of a hardware failure reason, however it was chosen to use these results anyway, which implies results obtained for this temperature should be considered with caution. 

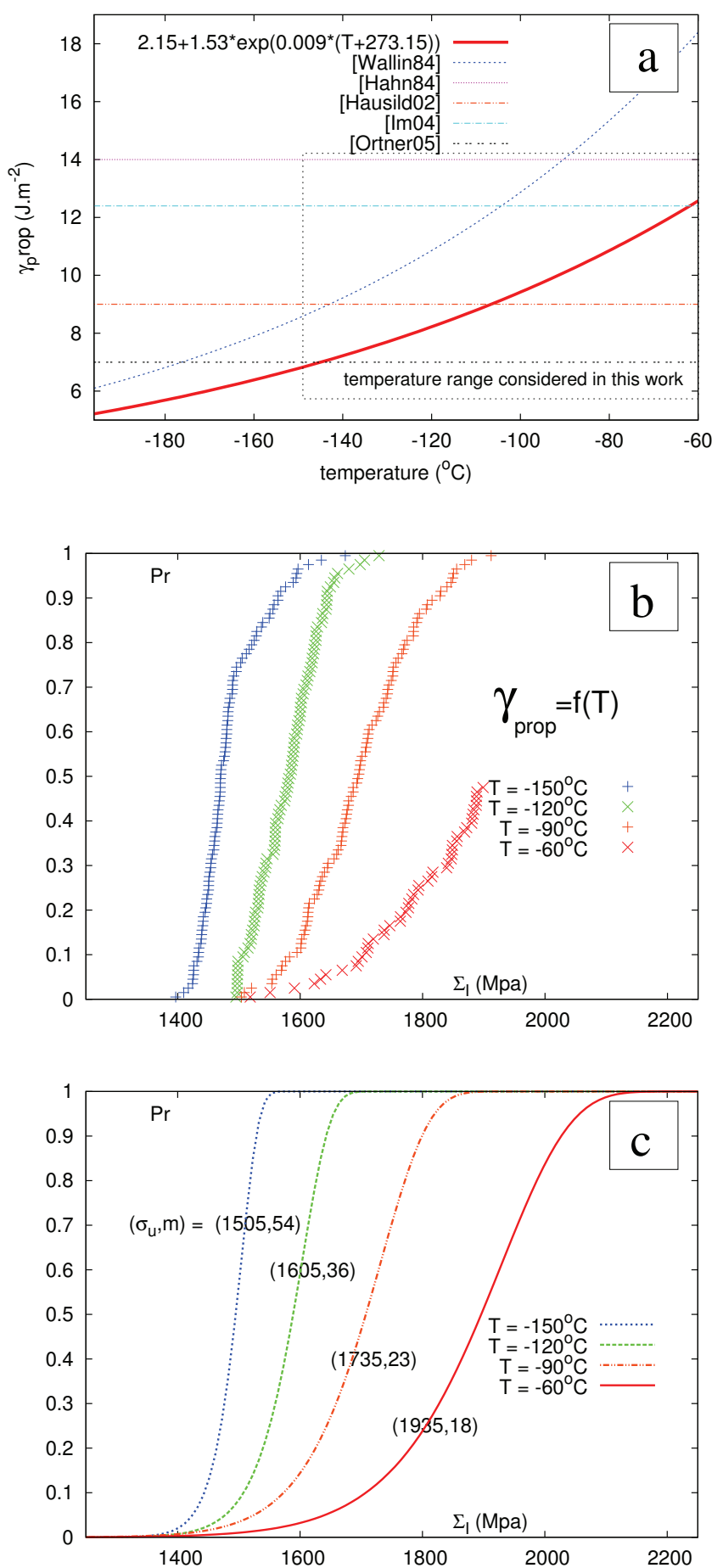

Fig. 15. Dependence of $\gamma_{\text {prop }}$ to temperature. a) Comparison of dependence used in this work with values found in literature.b) Computation results of simulation of fracture of the $\mathrm{EV}$ for this $\gamma_{\text {prop }}(T)$ value depending on temperature with respect to equation 26. c) Fitted Weibull parameters. 
In this case where ESE is considered as influenced by temperature, one can easily remark the dependence on temperature of the fitted Weibull parameters. Increase of temperature has an obvious effect on $\sigma_{u}$ parameter: since equation 26 expresses an increase of ESE, it also induces an increase in toughness of the material, which results globally in an increase of the mean stress to fracture in the EV. On the other hand, the effect on $m$ Weibull parameter is less intuitive. Again, it can be understood by observing the statistical repartition of the effective cleavage stress $\sigma_{c}^{\text {Max }}$ inside the EV for value of loading $\Sigma_{I}$ close to the mean calibrated $\sigma_{u}$ value. This is done in figure 16 .

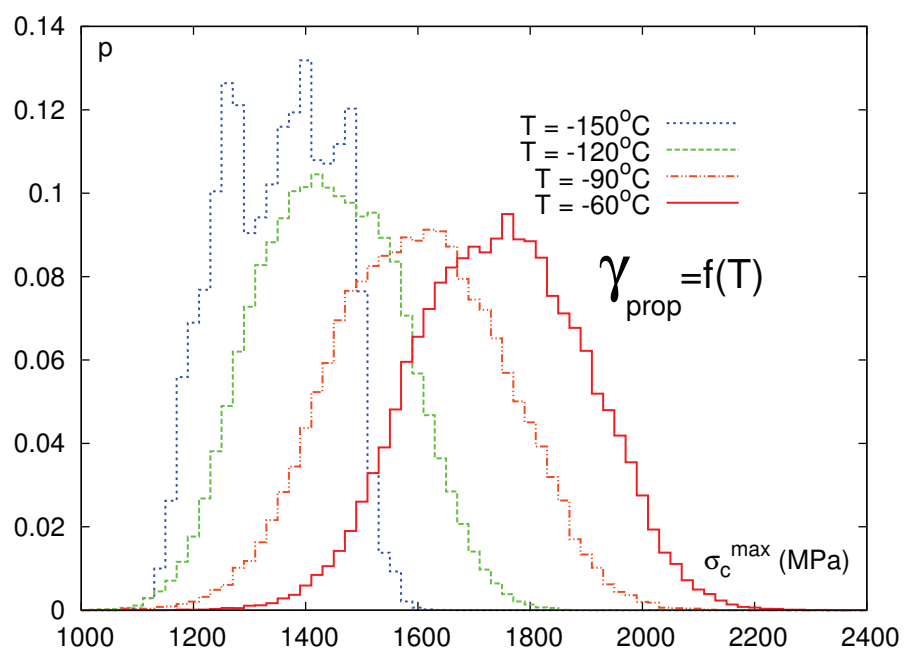

Fig. 16. Probability of the effective cleavage stress $\sigma_{c}^{\text {Max }}$ inside the EV for value of $\Sigma_{I}$ comparable to the identified $\sigma_{u}$ for triaxial loading at different temperatures.

The figure illustrates the fact that the increase in material toughness induced by temperature implies that more plasticity has to take place so that the tail of the $\sigma_{c}^{\text {Max }}$ distribution "raises" the $\sigma^{\text {grif }}$ distribution. In the same time, $\sigma_{c}^{M a x}$ distribution is enlarged so that the number of IP candidate to fulfill both fracture criteria increases too.

This result indicates that a simple temperature dependence of $\gamma_{\text {prop }}$ allows to capture a temperature dependence of Weibull parameters. One could even go further: it was shown in [2] that such a $\gamma_{\text {prop }}$ dependence applied only to the aggregate computation at $-150^{\circ} \mathrm{C}$ gives approximately the same dependence on Weibull parameters, which can be seen as a logical result when considering results presented in figure 14. This can be interpreted as the fact that if a temperature dependence is to be phenomenologically introduced in Weibull parameters for use in local approach, its physical interpretation may rely on lattice toughness evolution better than on macroscopic behaviour parameters evolution like the yield stress. 


\section{Discussion}

Hausild calibrated $m$ and $\sigma_{u}$ parameters with temperature experimentally for the same material [11], in a different temperature range $\left(\left[-90^{\circ} \mathrm{C} ;-30^{\circ} \mathrm{C}\right]\right)$. This inverse identification was made around the Ductile-Brittle Transition (DBT), taking into account the damage mechanisms. A comparison between these results and the results of the present work is made in figure 17. Other work presented similar results in the DBT [66].
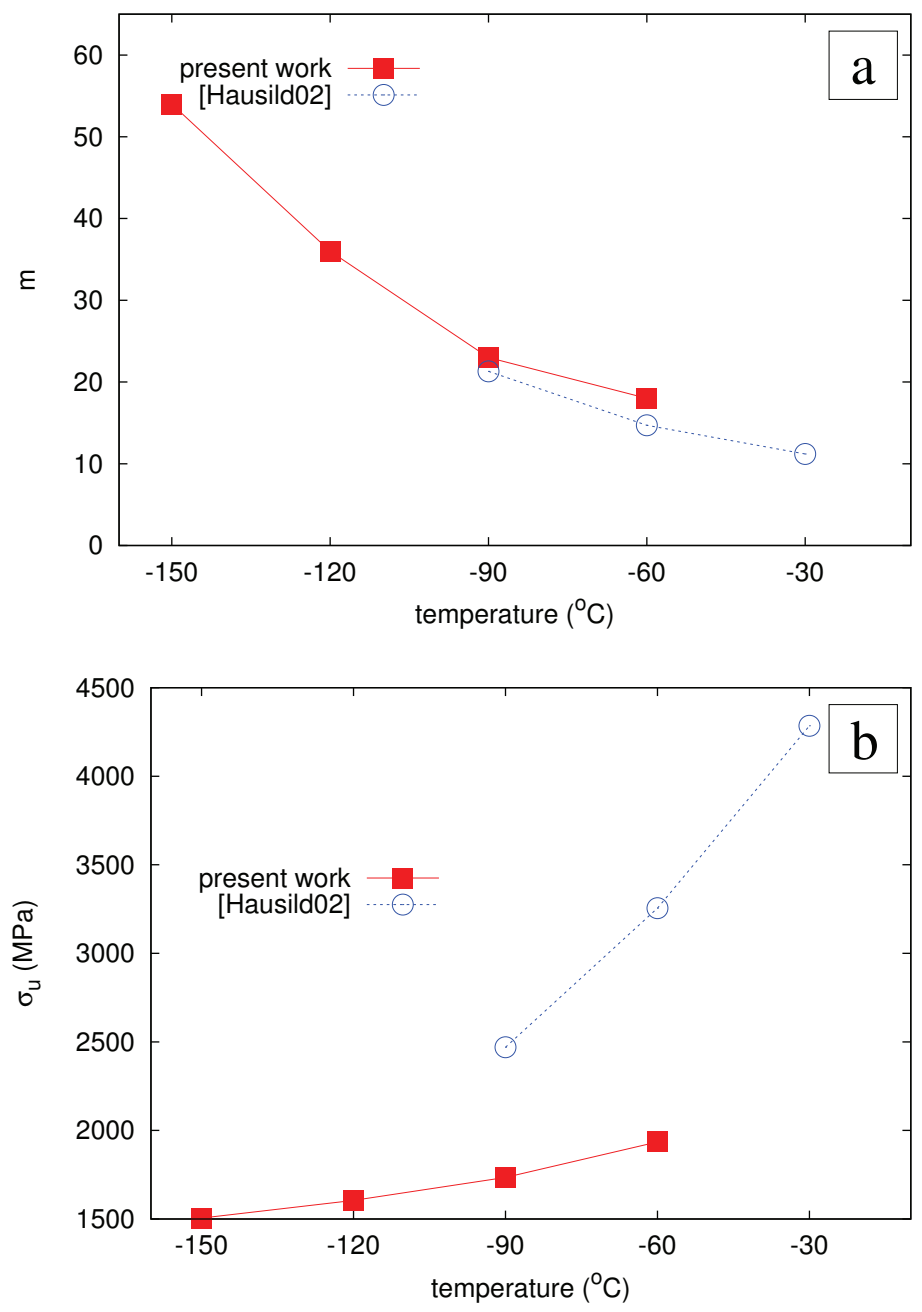

Fig. 17. Comparison of the Weibull parameters a) $m$ and b) $\sigma_{u}$ determined by the present micromechanical approach with Weibull parameters obtained in Hausild [11], and calibrated on experimental results in the DBT.

Evolutions of $m$ and $\sigma_{u}$ determined in the present work do not rely on any calibration on experimental work. So the agreement between $m$ values is quite notable. On the other hand, it is clear that $\sigma_{u}$ evolution estimated in this work does not fit with Hausild results, but at least, tendency is quite well respected 
(increase with temperature). Another remarkable point is that the agreement of this approach, which only considers brittle fracture of the EV, is obtained with an experimental fitting approach that consider damage mechanisms in the DBT. This may indicate that cleavage mechanisms considered and modeled in the present work remains valid in the DBT.

Another recently published work by Gao et al. investigates the loading rate effect on Weibull parameters[12]. It indicates that $\sigma_{u}$ decreases, and that $m$ remains constant. Results are not directly comparable to the present work, since a threshold value on Weibull stress $\sigma_{w-\min }$ was used in their work, but they confirm that changes in $\sigma_{u}$ in cases of temperature and strain rate evolution are of different nature: $m$ is affected in one case and not in the other one, although apparent changes in macroscopic yield stress are comparable. It is a clear evidence that macroscopic yield stress is not the only relevant parameter to describes those effects.

\section{Conclusion}

A micromechanical representation of plasticity has been set up in this paper, involving different scale representation. These scales were considered as appropriate since comparison to experimental characterization using X-ray diffraction after tensile straining at $-150^{\circ} \mathrm{C}$ shows that it intrinsically reproduces stress heterogeneities inside the ferritic matrix between different crystallographic orientations. This effect, captured without further calibration, has the advantage to allow access to intra-granular heterogeneities.

Based on this representation, micromechanical fracture criteria were used to compute time to fracture of an EV loaded with triaxial stress state at different low-temperatures, with realistic defects distributions, and for a volume consistent with the local approach. A cumulative probability of fracture for the EV was then computed, which demonstrates that the effect of temperature on Weibull parameters $m$ and $\sigma_{u}$ is to be linked with the evolution of the ESE $\gamma_{\text {prop }}$. Considering an evolution of ESE with temperature, experimentally fitted Weibull parameters evolution is reproduced, quite correctly for $m$, and with some limitations for $\sigma_{u}$, the tendency being correct. This is attributed to the deductive aspect of the approach that did not include inverse calibration in the fracture part. The defect distribution description and the ESE calibration were pointed out as the potential ways of improvement.

It is thought that modifying Beremin by transferring the effect of temperature identified in this work and the effect of stress triaxiality identified in previous one [26] to the EV fracture probability may improve results of local approach to fracture with different geometries tested at different temperature, while 
relying on micromechanical considerations.

\section{Acknowledgement}

EDF R\&D and EURATOM project PERFECT are greatfully acknowledged for financial support. G. Cailletaud and J. Besson from Ecole des Mines de Paris furnished ZeBuLoN FE code and technical support. R. Pesci, M. Wary, and D. Bouscaud from ENSAM de Metz are to be thanked about help on the experimental part. Dr. D. P. G. Lidbury, D. W. Beardsmore and S. Ortner supported this work by furnishing information about carbide sizes distribution within the RPV Mechanics sub-project collaborative work. J.-P. Mathieu wants to thank S. Bugat, who helped improving this manuscript with his cautious corrections.

\section{References}

[1] C. Zener, Fracturing of Metals, 29 th National Metal Congress and Exposition, American Society for Metals, 1948, pp. 3-31.

[2] J.-P. Mathieu, Analyse et modélisation micromécanique du comportement et de la rupture fragile de l'acier 16MND5 : Prise en compte des hétérogénéités microstructurales., Ph.D. thesis, Ecole Nationale Supérieure d'Arts et Métiers - avail. at http://pastel.paristech.org (2006).

[3] F. Beremin, A local criterion for cleavage fracture of a nuclear pressure vessel steel, Metall. Trans. A 14 (1983) 2277-2287.

[4] K. Wallin, T. Saario, K. Torronen, Statistical model for carbide induced brittle fracture in steels, Met. Sci. 18 (1984) 13-16.

[5] F. Mudry, A local approach to cleavage fracture, Nucl. Eng. and Design. 105 (1987) 65-76.

[6] K. Wallin, The scatter in K-results, Eng. Frac. Mech. 19 (1984) 1085-1093.

[7] S. Bordet, A. Kartensen, D. Knowles, C. Wiesner, A new statistical local criterion for cleavage fracture in steel. Part I : model presentation, Eng. Frac. Mech. 72 (2005) 435-452.

[8] S. Bordet, A. Kartensen, D. Knowles, C. Wiesner, A new statistical local criterion for cleavage fracture in steel. Part II : application to an offshore structural steel, Eng. Frac. Mech. 72 (2005) 453-474.

[9] M. Kroon, J. Faleskog, H. Osberg, A probabilistic model for cleavage fracture with a length scale - Parameter estimation and predictions of growing crack experiments, Eng. Frac. Mech. 75 (2008) 2398-2417. 
[10] B. Margolin, V. Shvetsovaa, A. Gulenkoa, V. Kostyleva, Prometey local approach to brittle fracture: Development and application, Eng. Frac. Mech. 75 (2008) 3483-3498.

[11] P. Hausild, Transition ductile-fragile dans un acier faiblement allié, Ph.D. thesis, Ecole Centrale de Paris (2002).

[12] X. Gao, J. Joyce, C. Roe, An investigation of the loading rate dependance of the Weibull stress parameters, Eng. Frac. Mech. 75 (2008) 1451-1467.

[13] X. Gao, G. Zhang, T. Srivatsan, A probabilistic model for prediction of cleavage fracture in the ductile to brittle transition region and the effect of temperature on models parameters, Mat. Sci. and Eng. A 415 (2006) 264-272.

[14] B. Tanguy, J. Besson, R. Piques, A. Pineau, Ductile to brittle transition of an A508 steel characterized by Charpy impact test - Part I : experimental results, Eng. Frac. Mech. 72 (2005) 49-72.

[15] B. Tanguy, J. Besson, R. Piques, A. Pineau, Ductile to brittle transition of an A508 steel characterized by Charpy impact test - Part I : modelling of the Charpy transition curve, Eng. Frac. Mech. 72 (2005) 413-434.

[16] T. Lin, A. Evans, Stochastic modelling of the independent roles of particle size and grain size in transgranular cleavage fracture, Metall. Trans. A 18 (1987) 641-651.

[17] K. Wallin, A. Laukkanen, New developments of the Wallin, Saario, Törrönen cleavage fracture model, Eng. Frac. Mech. 75 (2008) 3367-3377.

[18] M. Berveiller, A. Zaoui, Modeling of the plastic behavior of inhomogeneous media, J. Eng. Mat. Tech. 106 (1984) 295-298.

[19] G. Cailletaud, S. Forest, D. Jeulin, F. Feyel, I. Galliet, V. Mounoury, S. Quilici, Some elements of microstructural mechanics, Comput. Mat. Sci. 27 (2003) 351374.

[20] S. Bugat, Comportement et endommagement des aciers austéno-ferritiques vieillis : une approche micromécanique., Ph.D. thesis, Ecole des Mines de Paris (2000).

[21] O. Diard, S. Leclerq, G. Rousselier, G. Cailletaud, Evaluation of finite element based analysis of 3D multicristalline aggregates plasticity - Application to crystal plasticity model identification and the study of stress and strain fields near grain boundaries, Int. J. Plasticity 21 (2005) 691-722.

[22] R. Pesci, Etude micromécanique et caractérisation expérimentale du comportement et de l'endomagement de l'acier de cuve 16MND5 à basses températures, Ph.D. thesis, Ecole Nationale Supérieure d'Arts et Métiers - CER Metz (2004).

[23] J.-P. Mathieu, S. Berveiller, K. Inal, O. Diard, Plastic heterogeneities characterisation in 16MND5 RPV steel by X-Ray diffraction, comparison with finite-element approach, in: Proceedings of the 7th European Conference on Residual Stress, Trans Tech Publications, 2006, pp. 523-528. 
[24] J.-P. Mathieu, S. Berveiller, K. Inal, O. Diard, Multi-scale modelling of cleavage fracture at low temperatures : influence of heterogeneities at the granular scale, Fat. Fract. Eng. Mater. Struct. 29 (2006) 725-737.

[25] J.-P. Mathieu, S. Berveiller, K. Inal, O. Diard, Computation of brittle fracture local probability of a RPV bainitic steel based on realistic microstructure representation, in: Proceedings of the 9th European Mechanics of Materials Conference, Les Presses - Ecole des Mines, 2006, pp. 435-440.

[26] J.-P. Mathieu, S. Berveiller, K. Inal, O. Diard, Micromechanical modeling of brittle fracture of french RPV steel. a comprehensive study of the triaxiality effect, in: Proceedings of the ASME Pressure Vessels and Piping Conference 2008, ASME, 2008.

[27] H. Bhadeshia, R. Honeycombe, Steels - Microstructures and Properties, Butterworth-Heinemann, 2006.

[28] J. Mandel, Une généralisation de la théorie de la plasticité de W.T. Koiter, Int. J. Sol. Struct. 1 (1963) 273-295.

[29] L. Méric, G. Cailletaud, Single crystal modelling for Structural Calculations : Part 2 - F.E. Implementation, J. Eng. Mat. Tech. 113 (1991) 171-182.

[30] C. Petry, R. Pesci, Techniques expérimentales et mesures de champs. Applications l'acier de cuve 16MND5 - Technical report H-T24-2008-04403FR, EDF R\&D, 2009.

[31] T. Mori, K. Tanaka, Average stress in matrix and average elastic energy of materials with misfitting inclusions, Acta Metall. 21 (1973) 571-574.

[32] G. Cailletaud, P. Pilvin, Utilisation de modèles polycristallins pour le calcul par éléments finis, Rev. Européenne des Éléments Finis 3 (1994) 515-541.

[33] J. Besson, R. Foerch, Large scale object-oriented finite element code design, Comput. Meth. Appl. Mech. Eng. 142 (1997) 165-187.

[34] E. Gilbert, Random subdivision of space into crystals, Ann. Math. Stat. 33.

[35] L. Decker, D. Jeulin, Simulation 3D de matériaux aléatoires polycristallins, Rev. Métall./CIT Sc. et Tech. des Mat. (2000) 271-275.

[36] F. Barbe, Etude numérique de la plasticité d'aggrégats polycristallins, Ph.D. thesis, Ecole des Mines de Paris (2000).

[37] M. Umemoto, Z. Liu, K. Masuyama, K. Tsushiya, Production of bulk cementite and its characterization, Metall. and Mater. Trans. A, Phys. Metall. Mater. Science 32 (2001) 2127-2131.

[38] C. Schmitt, P. Lipinski, et al., Micromechanical modelling of the elastoplastic behavior of polycrystals containing precipitates - Application to hypo and hyper eutectoid steels, Int. J. Plasticity 13 (1997) 183-199.

[39] T. Hoc, S. Forest, Polycrystal modelling of IF-Ti steel under complex loading path, Int. J. Plasticity 17 (2001) 65-85. 
[40] T. Taoka, S. Takeuchi, E. Furubayashi, Slip systems and their critical shear stress in 3\% silicon iron, J. Phys. Soc. Japan 19 (1964) 701-711.

[41] A. Keh, Y. Nakada, Yielding, plastic flow and dislocation substructure in iron single crystals, Trans. JIM 9 (1968) 867-884.

[42] F. Barbe, S. Forest, G. Cailletaud, Intergranular and intragranular behavior of polycrystalline aggregates. Part 2 : Results, Int. J. Plasticity 17 (2001) 537-563.

[43] M. Libert, Etudes expérimentales et numérique de l'effet des mécanismesde plasticité sur la rupture fragile dans les aciers faiblement alliés., Ph.D. thesis, Ecole Centrale de Paris (2007).

[44] S. Queyreau, G. Monnet, B. Devincre, Slip systems interactions in $\alpha$-iron determined by dislocation dynamics simulations, Int. J. Plasticity 25 (2009) $361-377$.

[45] P. Kelly, Crystallography of lath martensite in steels., Acta Metall. 40 (1992) 1519-1530.

[46] N. Osipov, Génération et calcul de microstructures bainitiques, approche locale intra-granulaire de la rupture., Ph.D. thesis, Ecole des Mines de Paris (2007).

[47] R. Pesci, K. Inal, R. Masson, A crystallographic approach of brittle fracture in the 16MND5 bainitic steel. In situ X-Ray diffraction and scanning electron microscope measurements at low temperatures., Proceedings of 9th European Mecanichs of Materials Conference, 2006.

[48] A. Stroh, The formation of cracks as a result of plastic flow, Proc. Roy. Soc. London A 223 (1954) 404-414.

[49] A. Cottrell, Theory of Brittle Fracture in Steel and Similar Metals, Trans. of the Metall. Soc. of AIME 211 (1958) 192-203.

[50] J. Friedel, Dislocations, Pergamon Press Editions, 1964.

[51] E. Smith, The nucleation and growth of cleavage microcracks in mild-steels, Proceedings Conference on 'Physical basis of yield and fracture', Institute of Physics and Physical Society, Oxford London, 1966, pp. 36-46.

[52] G. Odette, T. Yamamoto, H. Rathburn, M. He, M. Hribernik, J. Rensman, Cleavage fracture and irradiation embrittlement of fusion reactor alloys : mechanisms, multiscale models, toughness measurements and implications to structural integrity assessment, J. of Nucl. Mat. 323 (2003) 313-340.

[53] Y.-R. Im, B.-J. Lee, Effect of microstructure on the cleavage fracture strength of low carbon Mn-Ni-Mo bainitic steels, J. of Nucl. Mat. 324 (2004) 33-40.

[54] B. Tanguy, Modélisation de l'essai charpy par l'approche locale de la rupture. application au cas de l'acier 16mnd5 dans le domaine de transition., Ph.D. thesis, Ecole des Mines de Paris (2001). 
[55] A. Echeverria, J. Rodriguez-Ibabe, Cleavage micromechanisms on microalloyed steels. Evolution with temperature of some critical parameters, Scripta Mater. 50 (2004) 307-312.

[56] S. Ortner, J. Duff, D. Beardsmore, Characterisation of Euro A Reference steel for application of $\mathrm{EOH}$ model of brittle fracture - Technical report SA/EIG/15234/R003 - Project PERFECT, SERCO Assurance, 2005.

[57] A. Griffith, The phenomena of rupture and flow in solids., Phil Trans. R. Soc. A 221 (1920) 163-198.

[58] J. Nohava, P. Hausild, M. Karlik, P. Bompard, Electron backscattering diffraction analysis of secondary cleavage cracks in a reactor pressure vessel steel, Mat. Charact. 49 (2003) 211-217.

[59] G. Hahn, The influence of microstructure on brittle fracture toughness, Metall. Trans. A 15 (1984) 947-959.

[60] X. Zhang, J. Knott, Cleavage fracture in bainitic and martensitic microstructures, Acta Mater. 47 (1999) 3483-3495.

[61] A. Lambert-Perlade, Rupture par clivage de microstructures d'aciers bainitiques obtenues en conditions de soudage., Ph.D. thesis, Ecole des Mines de Paris (2001).

[62] S. Lee, S. Kim, B. Hwang, B. Lee, C. Lee, Effect of carbide distribution on the fracture toughness in the transition temperature region of an SA 508 steel, Acta Mater. 50 (2002) 4755-4762.

[63] S. Ortner, Factor affecting the shape of the ductile-to-brittle transition, Int. J. of Press. Vessels and Piping 79 (2002) 693-700.

[64] B. Tanguy, J. Besson, A. Pineau, Comment on Effect of carbide distribution on the fracture toughness in the transition temperature region of an SA 508 steel, Scripta Mater. 49 (2003) 191-197.

[65] P. Blonski, A. Kiejna, Calculation of surface properties of BCC iron, Vacuum 74 (2004) 179-183.

[66] G. Bernauer, W. Brocks, W. Schmitt, Modification of the Beremin model for cleavage fracture in the transition region of a ferritic steel, Eng. Frac. Mech. 64 (1999) 305-325.

NOTICE : this is the author's version of a work that was accepted for publication in Journal of Nuclear Materials. Changes resulting from the publishing process, such as peer-review, editing, corrections, structural formatting, and other quality control mechanisms may not be reflected in this document. A definitive version was subsequently published in : Journal of Nuclear Materials, Volume 406, Issue 1, Pages 97-112. DOI : 10.1016/j.jnucmat.2010.02.025 